\title{
THE RUNGE-KUTTA LOCAL PROJECTION DISCONTINUOUS GALERKIN FINITE ELEMENT METHOD FOR CONSERVATION LAWS IV: THE MULTIDIMENSIONAL CASE
}

\author{
By \\ Bernardo Cockburn \\ Suchung Hou \\ and \\ Chi-Wang Shu
}

IMA Preprint Series \# 513

April 1989 


\title{
THE RUNGE-KUTTA LOCAL PROJECTION DISCONTINUOL'S GALERKIN FINITE ELEMENT METHOD FOR CONSERVATION LAWS IV: THE MULTIDIMENSIONAL CASE
}

\author{
BERNARDO COCKBURN†, SUCHUNG HOU† AND \\ CHI-WANG SHU $\ddagger$
}

\begin{abstract}
In this paper we study the two dimensional version of the Runge-Kutta Local-Projection Discontinuous Galerkin (RKDG) methods, already defined and analyzed in the one dimensional case. These schemes are defined on general triangulations. They can easily handle the boundary conditions, verify maximum principles, and are formally uniformly high order accurate. Preliminary numerical results showing the performance of the schemes on a variety of initial-boundary value problems are shown.
\end{abstract}

Key words. discontinuous finite elements, local projection, multidimensional conservation laws.

AMS(MOS) subject classifications. $65 \mathrm{M} 60,65 \mathrm{~N} 30,35 \mathrm{~L} 65$.

1. Introduction. This is the fourth article of a series in which we introduce, analyze and test numerically the RKDG-methods. These new numerical methods are devised to obtain approximations of the physically relevant solution of the initial boundary value problem associated with the hyperbolic conservation law

$$
\partial_{t} u+\operatorname{div} \mathbf{f}=0, \text { in }(0, T) \times \Omega,
$$

where $\Omega \subset \mathrm{R}^{d}, u=\left(u_{1}, \ldots, u_{m}\right)^{t}$, and $\mathbf{f}$ is such that any real combination of the Jacobian matrices $\sum_{i=1}^{d} \xi_{i} \frac{\partial f_{i}}{\partial u}$ has $m$ real eigenvalues and a complete set of eigenvectors. The case $d=1$ has been treated in [5],[6], and [7]. In [5] the idea of the method was introduced, and the model scheme, for which the approximate solution is taken to be piecewise linear in space, was studied in the framework of periodic boundary conditions. The resulting scheme was proven to be formally uniformly of order two, and to converge to a weak solution of (1.1). Numerical results showing the uniform second order accuracy as well as the convergence to the entropy solution in several cases were displayed. In [6] we extended these results to the general case, i.e., to the case of arbitrary boundary conditions and an approximate solution piecewisely a polynomial of degree $k$ in space. A local maximum principle, the TVBM (total variation bounded in the means) and the TVB (total variation bounded) properties, as well as convergence to a weak solution were proven. A formal uniform order of accuracy of $(k+1)$ was obtained, and was verified numerically for $k=1,2$ in several cases. Convergence to the entropy solution, as well as sharp capture

†School of Mathematics, University of Minnesota, Minneapolis, Minnesota 55455.

$\ddagger$ Division of Applied Mathematics, Brown University, Providence, Rhode Island 02912. 
of discontinuities, were also observed in these cases, even for non-convex fluxes $\mathbf{f}$. Finally, in [7] the schemes were extended to systems $(m>1)$, and numerical examples (showing the good performance of the methods for $k=1,2$ and $m=2,3$ ) were presented. Special attention was given to the Euler equations of gas dynamics. In this paper we shall extend our schemes to the multidimensional scalar case $(m=1, d>1)$. We thus complete (1.1a), with the initial condition:

$$
u(t=0)=u_{0}, \quad \text { in } \Omega
$$

where $u_{0} \in L^{\infty}(\Omega)$, and the boundary condition

$$
u=\gamma, \quad \text { in } .(0, T) \times \partial \Omega,
$$

where $\gamma \in L^{\infty}((0, T) \times \partial \Omega)$. See Bardos et al [1] for a correct interpretation of this condition. For the sake of clarity we shall restrict ourselves to the two-dimensional case, $d=2$.

When passing from the one dimensional case to the multidimensional case, the main challenge comes from the complicated geometry the domain $\Omega$ may have in practical applications. In this respect, finite element methods, such as the SUPG-method of Hughes and Brook [19], [20], [21], [22], [23], [24] (which has been analyzed by Johnson et al in [25], [26], and [27]), are better suited than finite difference methods, :ike for example the current versions of the ENO schemes [17],[18],[15], [16], [38], [39], or the Bell-Dawson-Shubin scheme [2]. This is the main reason for which the RKDG methods use a finite element discretization in space. The particular finite elements of our method allow an extremely simple treatment of the boundary conditions. No special numerical treatment of them is required in order to achieve uniform high order accuracy, as is the case for the finite difference schemes.

Another challenge is given by the increase of the complexity of the structure of the discontinuities. In the one dimensional case the Riemann problem can be solved in closed form and discontinuity curves in the $(x, t)$ plane are simple straight lines passing through the origin. However, in two dimensions only some special cases of the general Riemann problem have already been solved, and those display a very rich structure; see the works of Wagner [43], Lindquist [28], [29], and Tong [41], [42]. Thus, methods which allow triangulations that can be easily adapted to resolve this structure, should seriously be taken into consideration. Our methods allow extremely general triangulations. Moreover, the degree of the polynomial defining the approximate solution can be easily changed from element to element. Thus, adaptive versions of the RKDG methods can be easily defined, and shall constitute the subject of a forthcoming work.

From a theoretical point of view the passage from $d=1$ to $d=2$ is dramatic. In the one dimensional case it is possible to devise high order accurate schemes with the TVD (total variation diminishing) property, a property that implies the compactness of the sequence of 
approximate solutions generated by the schemes. (The TVD schemes were introduced by Harten [14], and a wide class of them was analyzed by Sweby [40]. Among these schemes we have, for example, (i) the MUSCL schemes, as analyzed by Osher [32], (ii) the schemes of Osher and Chacravarthy [33], (iii) the schemes obtained by a TVD time discretization introduced by Shu [37], and (iv) the quasi-monotone schemes introduced by Cockburn [8], [9], [10].) Unfortunatelly, in two dimensions, any TVD scheme is at most first order accurate. This interesting result was proven by Goodman and LeVeque [13]. In [10] it is shown under which conditions a quasi-monotone scheme (which is TVD) is formally high order accurate in two dimensions, but these conditions are quite restrictive. Thus, for $d=2$, there is a strong incompatibility between TVD-compactness and high order accuracy. We must emphasize however, that even in the one dimensional case these two properties are in conflict, for TVD schemes cannot be uniformly high order accurate: they are at most first order accurate at the critical points of the exact solution. This difficulty prompted the appearance of the so-called TVB (total variation bounded) schemes, which came out from rather different approaches. (Lucier [30] introduced a semidiscrete TVB scheme for which he proved an $L^{\infty}\left(0, T ; L^{1}\right)$-rate of convergence of $O\left(N^{-2}\right)$, where $N$ is the number of degrees of freedom defining the approximate solution. This result is true even if the exact solution has a finite number of discontinuities, a very surprising result that is now leading to even more surprising regularity results for the continuous problem; see [31]. Shu [35], [36], introduced a general technique to obtain uniformly high order accurate TVB schemes starting from TVD schemes; Sanders [34] introduced a third order accurate TVB scheme (which is TVD under another definition of total variation) whose accuracy degenerates to second order at critical points; the RKDG schemes are TVB schemes which are uniformly $k$-th order accurate, $k \geq 1,[5],[6],[7]$.) However, to prove the TVB property in two dimensions is a rather difficult task even for the simplest monotone scheme, if arbitrary triangulations are considered. On the other hand, maximum principles are not incompatible with high order accuracy, but it is well known that $L^{\infty}$-boundedness does not allow us to pass to the limit in the nonlinearity. Some kind of workable compactness criterion, one between TVB-compactness and $L^{\infty}$-boundedness, which is not in conflict with high order accuracy is still to be discovered and seems to be an interesting and challenging problem. Meanwhile, we shall content ourselves in obtaining maximum principles for the RKDG methods, a very desirable property in this kind of problems in which the values of the solution $u$ have physical meaning only in determined intervals. We remark that to the knowledge of the authors, no other class of schemes has a proven maximum principle for genearal nonlinearities $\mathbf{f}$, and arbitrary triangulations.

An outline of the paper follows. In Section 2 we present and analyze our schemes. The main result is the definition of the local projection $\Lambda \Pi$ for arbitrary triangulations, which does not destroy the high order accuracy of the scheme while enforcing a local maximum principle and allowing an extremely simple treatment of the boundary conditions. In Section 3 we present several numerical results, and in Section 4 we end with a summary 
and some concluding remarks.

\section{General Formulation.}

2.1 Preliminaries and notations. Let $\left\{t^{n}\right\}_{n=0}^{n t}$ be a partition of $[0, T]$. Set, as usual, $\Delta t^{n}=t^{n+1}-t^{n}, n=0, \ldots, n t-1$. Let us assume that the domain $\Omega$ is polygonal and let us denote by $\mathcal{T}_{h}$ a triangulation of $\Omega$. For the sake of simplicity we assume that if two elements of $\mathcal{T}_{h}$, say $K_{1}$ and $K_{2}$, are such that $e=K_{1} \cap K_{2} \neq \emptyset$ is either an edge of both $K_{1}$ and $K_{2}$ or a point.

We shall denote by $V_{h}$ the space of elements of $L^{\infty}(\Omega)$ whose restriction to $K \in \mathcal{T}_{h}$ belongs to a vector space $V(K)$. Note that the space $V(K)$ may be different for different elements $K$. The triangulation $\mathcal{T}_{h}$ of $\Omega$, induces a triangulation of $\partial \Omega$ that we shall denote by $\partial \mathcal{T}_{h}$. Let us denote by $\partial V_{h}$, the space of functions of $L^{\infty}(\partial \Omega)$ which are traces of functions of $V_{h}(\Omega)$.

As stated in [5], [6], and [7], to construct the RKDG methods we proceed as follows. First we discretize (1.1) in space using the Discontinuous Galerkin Method. The resulting equation can be put in ODE form as $\frac{d}{d t} u_{h}=L_{h}\left(u_{h}, \gamma_{h}(t)\right)$. Then, this ODE is discretized in time using the TVD Runge Kutta time discretization introduced in [38]. Finally, a local projection $\Lambda \Pi_{h}$ is applied to the intermediate values of the Runge Kutta discretization, in order to enforce stability. The general RKDG method has then the following form [7]:

(2.1a) Set $u_{h}^{0}=\Lambda \Pi_{h} \mathrm{P}_{V_{h}}\left(u_{0}\right)$;

(2.1b) For $n=0, \ldots, n t-1$ compute $u_{h}^{n+1}$ as follows:

(i) set $u_{h}^{(0)}=u_{h}^{n}$;

(ii) for $i=1, \ldots, k+1$ compute the intermediate functions:

$$
u_{h}^{(i)}=\Lambda \Pi_{h}\left\{\sum_{l=0}^{i-1} \alpha_{i l} u_{h}^{(l)}+\beta_{i l} \Delta t^{n} L_{h}\left(u_{h}^{(l)}, \gamma_{h}\left(t^{n}+d_{l} \Delta t^{n}\right)\right)\right\}
$$

(iii) set $u_{h}^{n+1}=u_{h}^{(k+1)}$;

where $\mathbf{P}_{V_{h}}$ is the $L^{2}$-projection into $V_{h}$, and $\gamma_{h}$ is the $L^{2}$-projection of $\gamma$ into $\partial V_{h}$. Note that this method is easy to code, for only a subroutine defining $L_{h}\left(u_{h}, \gamma_{h}(t)\right)$, and one defining $\Lambda \Pi_{h}$ are needed. Some Runge-Kutta time discretization parameters are displayed on the Table below, see [39]. 


\section{Table 1}

\begin{tabular}{|c|c|c|c|c|}
\hline \multicolumn{5}{|c|}{ Parameters of some practical Runge-Kutta time discretizations } \\
\hline order & $\alpha_{i l}$ & $\beta_{i l}$ & $d_{l}$ & $\max \left\{\alpha_{i l} / \beta_{i l}\right\}$ \\
\hline 2 & 1 & 1 & 0 & 1 \\
& $\frac{1}{2} \frac{1}{2}$ & $0 \frac{1}{2}$ & 1 & \\
\hline \multirow{3}{*}{3} & 1 & 1 & 0 & 1 \\
& $\frac{3}{4} \frac{1}{4}$ & $0 \frac{1}{4}$ & 1 & $\frac{1}{2}$ \\
\hline
\end{tabular}

In what follows we shall first describe in detail the operator $L_{h}$. Then we shall obtain conditions under which the RKDG methods verify maximum principles. The local projection $\Lambda \Pi_{h}$ will be then constructed in order to enforce those conditions. The stability and convergence properties of these schemes are summarized at the end of this Section.

2.2 The Operator $L_{h}$. In order to determine this operator we multiply (1.1a) by $v_{h} \in V_{h}$, integrate over $K \in \mathcal{T}_{h}$ and replace the exact solution $u$ by its approximation ${ }^{\prime \prime} h \in V_{h}$ :

$$
\frac{d}{d t} \int_{K} u_{h}(t, x) v_{h}(x) d x+\int_{K} \operatorname{div} \mathbf{f}\left(u_{h}(t, x)\right) v_{h}(x) d x=0, \forall v_{h} \in V_{h}
$$

Integrating by parts formally we obtain

$$
\begin{aligned}
\frac{d}{d t} \int_{K} u_{h}(t, x) v_{h}(x) d x & +\sum_{e \in \partial K} \int_{e} \mathbf{f}\left(u_{h}(t, x)\right) \cdot \mathbf{n}_{e, K} v_{h}(x) d \Gamma \\
& -\int_{K} \mathbf{f}\left(u_{h}(t, x)\right) \cdot \operatorname{grad} v_{h}(x) d x=0, \quad \forall v_{h} \in V_{h},
\end{aligned}
$$

where $\mathbf{n}_{e, K}$ is the outward unit normal to the edge $e$. Notice that $\mathbf{f}\left(u_{h}(t, x)\right) \cdot \mathbf{n}_{e, K}$ does not have a precise meaning, for $u_{h}$ is discontinuous at $x \in e \in \partial K$. Thus, as in the one dimensional case, we replace $\mathbf{f}\left(u_{h}(t, x)\right) \cdot \mathbf{n}_{e, K}$ by the function $h_{e, K}\left(u_{h}\left(t, x^{i n t(K)}\right), u_{h}\left(t, x^{e x t(K)}\right)\right)$ where:

$$
\begin{aligned}
& u_{h}\left(t, x^{i n t(K)}\right)= \lim _{y \rightarrow x} u_{h}(t, y), \\
& y \in K \\
& u_{h}\left(t, x^{e x t(K)}\right)= \begin{cases}\gamma_{h}(x, t), & \text { if } x \in \partial \Omega, \\
\lim _{\substack{y \rightarrow x \\
y \in(K)^{c}}} u_{h}(t, y), & \text { otherwise. }\end{cases}
\end{aligned}
$$


The function $h_{e, K}(\cdot, \cdot)$ is any function verifying the following conditions:

$(2.2 \mathrm{a})$

$$
h_{e, K}(u, u)=\mathbf{f}(u) \cdot \mathbf{n}_{e, K}
$$

$$
h_{e, K}(u, v) \text { is nondecreasing in } u \text { and nonincreasing in } v \text {, }
$$

$$
h_{e, K}(\cdot, \cdot) \text { is Lipschitz, }
$$

$$
h_{e, K}\left(u_{h}\left(x^{i n t(K)}\right), u_{h}\left(x^{e x t(K)}\right)\right)=-h_{e, K^{\prime}}\left(u_{h}\left(x^{i n t\left(K^{\prime}\right)}\right), u_{h}\left(x^{e x t\left(K^{\prime}\right)}\right)\right), K^{\prime} \cap K=e,
$$

i.e., it is a consistent two-point monotone Lipschitz flux, as defined in [11], consistent with $\mathbf{f}(u) \cdot \mathbf{n}_{e, K}$. The last property justifies $h_{e, K}(\cdot, \cdot)$ as a flux. Examples of $h_{e, K}$ can be found in [7, Remark 2.4]. Sometimes we shall write simply $h_{e, K}(t, x)$ instead of $h_{e, K}\left(u_{h}\left(t, x^{i n t(K)}\right), u_{h}\left(t, x^{e x t(K)}\right)\right)$.

In this way we obtain

$$
\begin{aligned}
& \frac{d}{d t} \int_{K} u_{h}(t, x) v_{h}(x) d x+\sum_{e \in \partial K} \int_{e} h_{e, K}(t, x) v_{h}(x) d \Gamma \\
& \cdot \quad-\int_{K} \mathbf{f}\left(u_{h}(t, x)\right) \cdot \operatorname{grad} v_{h}(x) d x=0, \quad \forall v_{h} \in V_{h} .
\end{aligned}
$$

Of course, we have to replace the integrals by quadrature rules that we shall choose as follows:

$$
\int_{e} h_{e, K}(t, x) v_{h}(x) d \Gamma \approx \sum_{i=1}^{L} \omega_{i} h_{e, K}\left(t, x_{e i}\right) v\left(x_{e i}\right)|e|
$$

$$
\int_{K} \mathbf{f}\left(u_{h}(t, x)\right) \cdot \operatorname{grad} v_{h}(x) d x \approx \sum_{j=1}^{M} \underline{\omega}_{j} \mathbf{f}\left(u_{h}\left(t, x_{K j}\right)\right) \cdot \operatorname{grad} v_{h}\left(x_{K j}\right)|K| .
$$

Thus, we finally obtain the weak formulation:

$$
\begin{aligned}
& \frac{d}{d t} \int_{k} u_{h}(t, x) v_{h}(x) d x+\sum_{e \in \partial K} \sum_{i=1}^{L} \omega_{i} h_{e, K}\left(t, x_{e i}\right) v\left(x_{e i}\right)|e| \\
& -\sum_{j=1}^{M} \underline{\omega}_{j} \mathbf{f}\left(u_{h}\left(t, x_{K j}\right)\right) \cdot \operatorname{grad} v_{h}\left(x_{K j}\right)|K|=0, \quad \forall v_{h} \in V_{h}, \quad \forall K \in \mathcal{T}_{h} .
\end{aligned}
$$


These equations can be rewritten in ODE form as $\frac{d}{d t} u_{h}=L_{h}\left(u_{h}, \gamma_{h}\right)$, where

$$
\begin{aligned}
& L_{h}: \mathcal{V}_{h} \times \partial \mathcal{V}_{h} \longrightarrow V_{h}, \\
& \frac{d}{d t}\left(u^{h}(t), v_{h}\right)=\left(L_{h}\left(u_{h}, \gamma_{h}\right), v_{h}\right), \quad \forall v_{h} \in V_{h}, \quad t \in(0, T),
\end{aligned}
$$

where $\mathcal{V}_{h}=\left\{w: \Omega \rightarrow \mathbb{R}:\left.w\right|_{K} \in \mathcal{C}^{0}(K), \quad \forall K \in \mathcal{T}_{h}\right\}, \partial \mathcal{V}_{h}=\{w: \partial \Omega \rightarrow \mathbb{R}:$ $\left.\left.w\right|_{e} \in \mathcal{C}^{0}(e), \quad \forall e \in \partial \mathcal{T}_{h}\right\}$, and $(\cdot, \cdot)$ is the usual $L^{2}(\Omega)$ inner product. Notice that in order to go from the weak form (2.4) to the ODE (2.5b) a matrix has to be inverted. However, this can be easily done by hand, for its order is equal to the dimension of the local space $V(K)$. It is also important to remark that any choice of the degrees of freedom of the approximate solution is allowed in this formulation.

Thus, the operator $L_{h}\left(u_{h}, \gamma_{h}\right)$ is a discrete approximation of $-\operatorname{div} \mathbf{f}(u)$ (together with the corresponding boundary conditions!). The following result gives an indication of the quality of this approximation.

Proposition 2.1. Let $\mathbf{f}(u) \in W^{k+2, \infty}(\Omega)$, and set $\gamma=\operatorname{trace}(u)$. Let the quadrature rule over the edges be exact for polynomials of degree $(2 k+1)$, and let the one over the element be exact for polynomials of degree $(2 k)$. Assume that the family of triangulations $\mathcal{F}=\left\{\mathcal{T}_{h}\right\}_{h>0}$ is regular, i.e., that there is a constant $\sigma$ such that:

$$
\frac{h_{K}}{\rho_{K}} \geq \sigma, \quad \forall K \in \mathcal{T}_{h}, \quad \forall \mathcal{T}_{h} \in \mathcal{F},
$$

where $h_{K}$ is the diameter of $K$, and $\rho_{K}$ is the diameter of the biggest ball included in $K$. Then, if $V(K) \supset P^{k}(K), \quad \forall K \in \mathcal{T}_{h}$ :

$$
\left\|L_{h}(u, \gamma)+\operatorname{div} \mathbf{f}(u)\right\|_{L^{\infty}(\Omega)} \leq C h^{k+1}|\mathbf{f}(u)|_{W^{k+2, \infty}(\Omega)} .
$$

We are, of course, assuming implicitly that the dimension of the local space $V(K)$ is uniformly bounded from above. A $p$-version of these schemes can certainly be considered, but we shall leave this as the subject of future work.

For the proof we shall need the following direct consequence of the Bramble-Hilbert Lemma, [4]. Then,

LEMmA 2.2. Let $E_{\Omega}(\phi)=\int_{\Omega} \phi-\sum_{l=1}^{L} \omega_{l} \phi\left(x_{l}\right)|\Omega|$, and suppose that $E_{\Omega}(\phi)=0, \forall \phi \in P^{r}(\Omega)$.

$$
\left|E_{\Omega}(g \psi)\right| \leq C|\Omega| h^{r+1-s}|\psi|_{L^{\infty}}(\Omega)|g|_{W^{r+1-s, \infty}(\Omega)}, \quad \forall \psi \in P^{s}(\Omega),
$$

where $h=\operatorname{diam} \Omega$.

Proof (of Proposition 2.1). We have

$$
\left\|L_{h}(u, \gamma u)+\operatorname{div} \mathbf{f}(u)\right\|_{L^{\infty}(K)} \leq e_{1}+e_{2},
$$


where

$$
\begin{aligned}
& e_{1}=\| \operatorname{div} \mathbf{f}\left(u ;-\mathbf{P}_{P^{k}(K)}(\operatorname{div} \mathbf{f}(u)) \|_{i i^{\infty}(K)},\right. \\
& e_{2}=\left\|L_{h}(u, \gamma)+\mathbf{P}_{P^{k}(K)}(\operatorname{div} \mathbf{f}(u))\right\|_{L^{\infty}(K)},
\end{aligned}
$$

and $\mathbf{P}_{P^{k}(K)}$ is the $L^{2}$-projection into $P^{k}(K)$. Using the regularity of the triangulation $\mathcal{T}_{h}$ we obtain, by a well known approximation theory result, [4], that:

$$
e_{1} \leq C h^{k+1}|\operatorname{div} \mathbf{f}(u)|_{W(K)^{k+1, \infty}} .
$$

To estimate $e_{2}$ we proceed as follows. Taking into account the definition of $L_{h},(2.5)$, (2.4), we have, for $v_{h} \in P^{k}(K)$ :

$$
\begin{aligned}
\Theta\left(v_{h}\right)= & \frac{1}{|K|} \int_{K}\left(L_{h}(u, \gamma)+\mathbf{P}_{P^{k}(K)}(\operatorname{div} \mathbf{f}(u))\right) v_{h} d x \\
= & \frac{1}{|K|} \int_{K}\left(L_{h}(u, \gamma)+\operatorname{div} \mathbf{f}(u)\right) v_{h} d x \\
= & \frac{1}{|K|} \int_{K} L_{h}(u, \gamma) v_{h} d x \\
& +\frac{1}{|K|} \sum_{e \in \partial K} \int_{e} \mathbf{f}(u(t, x)) \cdot \mathbf{n}_{e, K} v_{h}(x) d \Gamma-\frac{1}{|K|} \int_{K} \mathbf{f}\left(u_{h}(x, t)\right) \cdot \operatorname{grad} v_{h}(x) d x \\
= & \frac{1}{|K|} \sum_{e \in \partial K} E_{e}\left(\mathbf{f}(u) \cdot \mathbf{n}_{e, K} v_{h}\right)-\frac{1}{|K|} E_{K}\left(\mathbf{f}(u) \cdot \operatorname{grad} v_{h}\right),
\end{aligned}
$$

where

$$
\begin{gathered}
E_{e}(\phi)=\int_{e} \phi-\sum_{i=1}^{L} \omega_{i} \phi\left(x_{e i}\right)|e| \\
E_{K}(\phi)=\int_{K} \phi-\sum_{j=1}^{M} \underline{\omega}_{j} \phi\left(x_{K j}\right)|K| .
\end{gathered}
$$

But,

$$
\begin{aligned}
\frac{1}{|K|}\left|E_{e}\left(\mathbf{f}(u) \cdot \mathbf{n}_{e, K} v_{h}\right)\right| \leq & C \frac{|e|}{|K|} h^{k+2}\left|\mathbf{f}(u) \cdot \mathbf{n}_{e, K}\right|_{W^{k+2, \infty}(e)}\left|v_{h}\right|_{L^{\infty}(K)} \\
& \text { by hypothesis and Lemma } 2.2 \\
\leq & C h^{k+1}|\mathbf{f}(u)|_{W^{k+2, \infty}(K)}\left|v_{h}\right|_{L^{\infty}(K)} \\
& \text { by the regularity of the triangulation, }
\end{aligned}
$$


and similarly,

$$
\frac{1}{|K|}\left|E_{K}\left(\mathbf{f}(u) \cdot \operatorname{grad} v_{h}\right)\right| \leq C h^{k+1}|\mathbf{f}(u)|_{W^{k+2, \infty}(K)}\left|v_{h}\right|_{L^{\infty}(K)}
$$

The result follows by setting $v_{h}=L_{h}(u, \gamma)+\mathbf{P}_{V_{h}}(\operatorname{div} \mathbf{f}(u))$, and using the inequality

$$
\left|w_{h}\right|_{L^{\infty}(K)}^{2} \leq C \frac{1}{|K|}\left|w_{h}\right|_{L^{2}(K)}^{2}
$$

which is valid if $w_{h} \in V(K)$. (Notice that all the norms are equivalent in finite dimensional spaces; the factor $1 /|K|$ follows from a standard scaling argument, see [4].) The constant $C$ depends solely on the dimension of $V(K)$ which was implicitly assumed to be uniformly bounded.

2.3. The quest of a maximum principle. We now consider the problem of rendering our schemes $L^{\infty}$-stable. As we said earlier we shall construct a local projection $\Lambda \Pi_{h}$ whose task will be to enforce a maximum principle on them. The fact that this is indeed possible is to a great extent due to the form of the time discretization technique, as we show below. Let us write $u_{h}$ as $\bar{u}_{h}+\tilde{u}_{h}$, where $\bar{u}_{h}$ is piecewise constant and $\tilde{u}_{h}$ has zero-mean in each element $K$. The restriction of $\bar{u}_{h}$ to the element $K$ will be denoted by $\bar{u}_{K}$.

LEMMA 2.3. Let the coefficients $\alpha_{i l}$ of the Runge-Kutta time discretization be positive and such that $\sum_{l=0}^{i-1} \alpha_{i l}=1$, for $i=1, \cdots, k+1$. Set $w_{h}=u_{h}+\delta \Delta t^{m} L_{h}\left(u_{h}, \gamma_{h}\right)$, and suppose that for $c f l \in\left[0, c f l_{0} /|\delta|\right]$, where,

$$
\begin{aligned}
c f l & =\sup _{n=1, \cdots, n t ; e \in \partial K ; K \in \mathcal{T}_{h}} \Delta t^{n} \frac{|e|}{|K|}\left\|\mathbf{f}^{\prime} \cdot \mathbf{n}_{e, K}\right\|_{L^{\infty}\left[a_{0}, b_{0}\right]}, \\
a_{0} & =i n f_{x \in \Omega, t \in\left(0, t^{n+1}\right), y \in \partial \Omega}\left\{u_{o}(x), \gamma(t, y)\right\} \\
b_{0} & =\sup _{x \in \Omega, t \in\left(0, t^{n+1}\right), y \in \partial \Omega}\left\{u_{o}(x), \gamma(t, y)\right\}
\end{aligned}
$$

the following maximum principle is verified:

$$
\bar{u}_{h}, \gamma_{h} \in[a, b] \Rightarrow \bar{w}_{h} \in\left[a-M h^{2}, b+M h^{2}\right]
$$

where $M$ is some nonnegative parameter. Then, if $c f l \in\left[0, c f l_{0} /\left|\max _{i, l}\left\{\mid \frac{\beta_{i l}}{\alpha_{i l}}\right\}\right|\right]$,

$$
\bar{u}_{h}^{n} \in\left[a_{0}-(k+1) n M h^{2}, b_{0}+(k+1) n M h^{2}\right]
$$

Proof. We proceed by induction. Assume that

$$
\bar{u}_{h}^{m} \in\left[a_{0}-(k+1) m M h^{2}, b_{0}+(k+1) m M h^{2}\right], \quad \text { for } m=0, \cdots, n \text {. }
$$


The case $m=0$ is trivially verified. Let us prove that it is also verified for $m=n+1$. Set $[a, b]=\left[a_{0}-(k+1) m M h^{2}, b_{0}+(k+1) m M h^{2}\right]$. We claim that $\bar{u}_{h}^{(l)} \in\left[a-l M h^{2}, b+l M h^{2}\right]$ for $j=0, \cdots, k+1$. This is true for $l=0$, for $u_{h}^{(0)}=u_{h}^{n}$ by (2.ib) (ii). Assume it is true for $l=0, \cdots, i-1$. Then, by the definition $(2.1 \mathrm{~b})$ of the intermediate function $u_{h}^{(i)}$ :

$$
\begin{aligned}
u_{h}^{(i)} & =\sum_{l=0}^{i-1} \alpha_{i l} u_{h}^{(l)}+\beta_{i l} \Delta t^{n} L_{h}\left(u_{h}^{(l)}, \gamma_{h}\left(t^{n}+d_{l} \Delta t^{n}\right)\right), \\
& =\sum_{l=0}^{i-1} \alpha_{i l}\left\{u_{h}^{(l)}+\frac{\beta_{i l}}{\alpha_{i l}} \Delta t^{n} L_{h}\left(u_{h}^{(l)}, \gamma_{h}\left(t^{n}+d_{l} \Delta t^{n}\right)\right)\right\} .
\end{aligned}
$$

This implies that

$$
\begin{aligned}
\bar{u}_{h}^{(i)} & \in \sum_{l=0}^{i-1} \alpha_{i l}\left[a-j M h^{2}, b+j M h^{2}\right] \\
\subset & \subset\left\{\sum_{l=0}^{i-1} \alpha_{i l}\right\}\left[a-i M h^{2}, b+i M h^{2}\right] \\
& =\left[a-i M h^{2}, b+i M h^{2}\right] .
\end{aligned}
$$

But, by (2.1b)(iii) $u_{v_{l}}^{n+1}=u_{h}^{(k+1)}$, and so $\bar{u}_{h}^{n+1}=\bar{u}_{h}^{(k+1)} \in\left[a-(k+1) M h^{2}, b+(k+1) M h^{2}\right]$. This completes the proof.

In this way, the existence of a maximum principle for the RKGD schemes is reduced to the existence of the maximum principle (2.8). Thus, to construct the projection $\Lambda \Pi_{h}$ we first study the conditions (on $u_{h}$ ) under which the maximum principle (2.8) holds, and then we define $\Lambda \Pi$ in order to enforce them. This is the approach we took in the one dimensional scalar case, and is the same we shall take in this case. Next, we study those conditions. The very construction of $\Lambda \Pi_{h}$ will be considered in the next section.

If $e \in \partial K$ let us denote by $K_{e}$ the element such that $K \cap K_{e}=e$. In this way, if $x_{e l}$ is the $l$-th point of the quadrature rule $(2.2 \mathrm{a})$ on the edge $e$, we shall write

$$
\begin{aligned}
& u_{h}\left(x_{e l}^{i n t(K)}\right)=u_{K, e l}=\bar{u}_{K}+\tilde{u}_{K, e l}, \\
& u_{h}\left(x_{e l}^{e x t(K)}\right)=u_{K_{e}, e l}=\bar{u}_{K_{e}}+\tilde{u}_{K_{e}, e l}, \\
& \gamma_{h}\left(x_{e l}\right)=\gamma_{e l} .
\end{aligned}
$$

Set $w_{h}=u_{h}+\delta^{\prime} L_{h}\left(u_{h}, \gamma_{h}\right)$, where $\delta^{\prime}=\Delta t^{m} \cdot \delta$. Thus, by the definition of $L_{h}\left(u_{h}, \gamma_{h}\right)$ $(2.4),(2,5)$ :

$\bar{w}_{K}=\bar{u}_{K}-\left\{\sum_{e \in \partial K \backslash \partial \Omega} \sum_{l=1}^{L}\left[\frac{\delta^{\prime} \omega_{l}|e|}{|K|}\right] h_{e, K}\left(u_{K, e l}, u_{K_{e}, e l}\right)\right\}-\left\{\sum_{e \in \partial K \cap \partial \Omega} \sum_{l=1}^{L}\left[\frac{\delta^{\prime} \omega_{l}|e|}{|K|}\right] h_{e, K}\left(u_{K, e l}, \gamma_{e l}\right)\right\}$. 
Noting that

$$
\sum_{e \in \partial K} \sum_{l=1}^{L}\left[\frac{\delta^{\prime} \omega_{l}|e|}{|K|}\right]_{e, K}\left(\bar{u}_{K}, \bar{u}_{\bar{K}}\right)=\frac{\delta^{\prime}}{|K|} \int_{\partial K} \mathbf{f}\left(\bar{u}_{K}\right) \cdot \mathbf{r}_{\mathbf{i}}=0
$$

we can write:

$$
\begin{aligned}
& \bar{w}_{K}=\bar{u}_{K}-\left\{\sum_{e \in \partial K \backslash \partial \Omega} \sum_{l=1}^{L}\left[\frac{\delta^{\prime} \omega_{l}|e|}{|K|}\right]\left(h_{e, K}\left(u_{K, e l}, u_{K_{e}, e l}\right)-h_{e, K}\left(\bar{u}_{K}, \bar{u}_{K}\right)\right)\right\} \\
&-\left\{\sum_{e \in \partial K \cap \partial \Omega} \sum_{l=1}^{L}\left[\frac{\delta^{\prime} \omega_{l}|e|}{|K|}\right]\left(h_{e, K}\left(u_{K, e l}, \gamma_{e l}\right)-h_{e, K}\left(\bar{u}_{K}, \bar{u}_{K}\right)\right)\right\} \\
&= \bar{u}_{K}-\left\{\sum _ { e \in \partial K \backslash \partial \Omega } \sum _ { l = 1 } ^ { L } [ \frac { \delta ^ { \prime } \omega _ { l } | e | } { | K | } ] \left\{\left(h_{e, K}\left(u_{K, e l}, u_{K_{e}, e l}\right)-h_{e, K}\left(\bar{u}_{K}, u_{K_{e}, e l}\right)\right)\right.\right. \\
&\left.\left.+\left(h_{e, K}\left(\bar{u}_{K}, u_{K_{e}, e l}\right)-h_{e, K}\left(\bar{u}_{K}, \bar{u}_{K}\right)\right)\right\}\right\} \\
&-\left\{\sum _ { e \in \partial K \cap \partial \Omega } \sum _ { l = 1 } ^ { L } [ \frac { \delta ^ { \prime } \omega _ { l } | e | } { | K | } ] \left\{\left(h_{e, K}\left(u_{K, e l}, \gamma_{e l}\right)-h_{e, K}\left(\bar{u}_{K}, \gamma_{e l}\right)\right)\right.\right. \\
&\left.\left.+\left(h_{e, K}\left(u_{K}, \gamma_{e l}\right)-h_{e, K}\left(\bar{u}_{K}, \bar{u}_{K}\right)\right)\right\}\right\} .
\end{aligned}
$$

Using the fact that the one-dimensional flux $h_{e, x_{K}}$ is Lipschitz we obtain:

$$
\begin{aligned}
\bar{w}_{K}=\bar{u}_{K} & -\left\{\sum_{e \in \partial K \backslash \partial \Omega} \sum_{l=1}^{L}\left[\frac{\delta^{\prime} \omega_{l}|e|}{|K|}\right]\left\{h_{e l, 1}\left\{\tilde{u}_{K, e l}\right\}+h_{e l, 2}\left(\left\{\bar{u}_{K_{e}}-\bar{u}_{K}\right\}+\left\{\tilde{u}_{K_{e}, e l}\right\}\right)\right\}\right\}+ \\
& -\left\{\sum_{e \in \partial K \backslash \partial \Omega} \sum_{l=1}^{L}\left[\frac{\delta^{\prime} \omega_{l}|e|}{|K|}\right]\left\{h_{e l, 1}\left\{\tilde{u}_{K, e l}\right\}+h_{e l, 2}\left(\left\{\gamma_{e l}-\bar{u}_{K}\right\}\right)\right\}\right\} \\
=\bar{u}_{K} & +\left\{\sum_{e \in \partial K \backslash \partial \Omega} \sum_{l=1}^{L}\left[-\frac{\delta^{\prime} \omega_{l}|e|}{|K|} h_{e l, 2}\right]\left\{\bar{u}_{K_{e}}-\bar{u}_{K}\right\}+\sum_{e \in \partial K \cap \partial \Omega} \sum_{l=1}^{L}\left[-\frac{\delta^{\prime} \omega_{l}|e|}{|K|} h_{e l, 2}\right]\left\{\gamma_{e l}-\bar{u}_{K}\right\}\right\} \\
& +\left\{\sum_{e \in \partial K} \sum_{l=1}^{L}\left[\frac{\delta^{\prime} \omega_{l}|e|}{|K|} h_{e l, 1}\right]\left\{-\tilde{u}_{K, e l}\right\}+\sum_{e \in \partial K \backslash \partial \Omega} \sum_{l=1}^{L}\left[-\frac{\delta^{\prime} \omega_{l}|e|}{|K|} h_{e l, 2}\right]\left\{\tilde{u}_{K_{e}, e l}\right\}\right\} .
\end{aligned}
$$

If we assume that $\omega_{l} \geq 0$, then the quantities between brackets are nonnegative numbers, by (2.2b), which are bounded from above, thanks to (2.2c) and (2.2a). Now, if the quantities $-\tilde{u}_{K, e l}$ and $\tilde{u}_{K_{e}, e l}$ can be written as positive linear combinations of $\bar{u}_{K_{e}}-\bar{u}_{K}$, and $\bar{\gamma}_{e}-\bar{u}_{K}$, i.e., if:

$$
\begin{aligned}
-\tilde{u}_{K, e l} & =\sum_{d \in \partial K \backslash \partial \Omega} \theta_{K, e l d}\left(\bar{u}_{K_{d}}-\bar{u}_{K}\right)+\sum_{d \in \partial K \cap \partial \Omega} \theta_{K, \text { eld }}\left(\bar{\gamma}_{d}-\bar{u}_{K}\right), \\
\tilde{u}_{K_{e}, e l} & =\sum_{d \in \partial K \backslash \partial \Omega} \eta_{K_{e}, e l d}\left(\bar{u}_{K_{d}}-\bar{u}_{K}\right)+\sum_{d \in \partial K \cap \partial \Omega} \eta_{K_{e}, e l d}\left(\bar{\gamma}_{d}-\bar{u}_{K}\right),
\end{aligned}
$$


where

$$
\theta_{K, e l d}, \quad \eta_{K_{e}, e l d} \geq 0
$$

and

$$
\bar{\gamma}_{e}=\sum_{\ell=1}^{L} \omega_{\ell} \gamma_{e l}
$$

then

$$
\bar{w}_{K}=\bar{u}_{K}-\delta \Delta t^{m} \cdot\left\{\sum_{e \in \partial K \backslash \partial \Omega} \Theta_{e}\left\{\bar{u}_{K_{e}}-\bar{u}_{K}\right\}+\sum_{e \in \partial K \cap \partial \Omega} \sum_{l=1}^{L} \Theta_{e l}\left(\gamma_{e l}-\bar{u}_{K}\right)\right\}
$$

where

$$
\begin{aligned}
& \Theta_{e}=\sum_{\ell=1}^{L} \frac{\omega_{\ell}|e|}{|K|}\left[-h_{e l, 2}\right]+\sum_{d \in \partial K} \sum_{\ell=1}^{L} \frac{\omega_{\ell}|d|}{|K|}\left[h_{d \ell, 1}\right] \theta_{K, d \ell e} \\
&+\sum_{d \in \partial K \backslash \partial \Omega} \sum_{\ell=1}^{L} \frac{\omega_{\ell}|d|}{|K|}\left[h_{d \ell, 2}\right] \eta_{K_{d}, d \ell e} \\
& \Theta_{e l}=\frac{\omega_{l}|e|}{|K|}\left[-h_{e l, 2}\right]+\omega_{l} \sum_{d \in \partial K} \sum_{\ell=1}^{L} \frac{\omega_{\ell}|d|}{|K|}\left[h_{d \ell, 1}\right] \theta_{K, d \ell e} \\
&+\omega_{l} \sum_{d \in \partial K \backslash \partial \Omega} \sum_{\ell=1}^{L} \frac{\omega_{\ell}|d|}{|K|}\left[h_{d \ell, 2}\right] \eta_{K_{d}, d \ell e} .
\end{aligned}
$$

Thus, if

$$
\sum_{e \in \partial K \backslash \partial \Omega} \Theta_{e}+\sum_{e \in \partial K \cap \partial \Omega} \sum_{l=1}^{L} \Theta_{e l} \leq 1 / \delta
$$

then

$$
\bar{w}_{K} \in I\left(\bar{u}_{K} ; \bar{u}_{K_{e}}, e \in \partial K \backslash \partial \Omega ; \gamma_{e l}, e \in \partial K \cap \partial \Omega, l=1, \cdots, L\right)
$$

where $I\left(a_{1}, \ldots, a_{n}\right)=\left[\min \left\{a_{1}, \ldots, a_{n}\right\}, \max \left\{a_{1}, \ldots, a_{n}\right\}\right]$. This is the local maximum principle we were looking for. Let us summarize this result as follows: 
Proposition 2.4. Assume that all the weights of the quadrature rule (2.3a), $\omega_{l}$, are nonnegative. Set $w_{h}=u_{h}+\delta \cdot \Delta t^{m} \cdot L_{h}\left(u_{h}, \gamma_{h}\right)$, where $u_{h}$ satisfies conditions (2.9). Then, the maximum principle (2.12) is satisfied for the elements $K \in \mathcal{T}_{h}$ for which the conaicion (2.11)-(2.10) is verified.

2.4. The $\Lambda \Pi_{h}$ projection. We now prove that conditions (2.9) can actually be satisfied without compromising the order accuracy of the method, provided a class of (very general) triangulations is used (the so-called B-triangulations), and give a sensible rule for computing the coefficients $\theta_{K, \text { eld }}$ and $\eta_{K_{e}, \text { eld }}$. Then, we define a class of local projections $\Lambda \Pi_{h}$ which enforce conditions (2.9) under a suitable $c f l$-condition which results from condition (2.11)-(2.10).

Pick an element of the triangulation $\mathcal{T}_{h}, K$ say. We associate it with the vectors

$$
\mathbf{d}_{K, e}= \begin{cases}\mathbf{B}_{e}-\mathbf{B}, & \text { if } e \notin \partial \Omega, \\ \mathbf{M}_{e}-\mathbf{B}, & \text { if } e \in \partial \Omega\end{cases}
$$

where $\mathrm{B}$ denotes the barycenter of $K, \mathrm{~B}_{e}$ denotes the one of $K_{e}$, and $\mathrm{M}_{e}$ denotes the middle point of the edge $e$. To each point $x_{e \ell}$ of the quadrature rule (2.2a) we associate the vectors

$$
\begin{aligned}
\mathrm{d}_{K, e \ell} & =x_{e \ell}-\mathrm{B}, \\
\mathrm{d}_{K_{e}, e \ell} & =x_{e \ell}-\mathrm{B}_{e},
\end{aligned}
$$

and express them as linear combinations of the vectors $\mathrm{d}_{K, e}$ as follows:

$$
\begin{aligned}
-\mathbf{d}_{K, e \ell} & =\theta_{K, e \ell e_{1}} \mathbf{d}_{K, e_{1}}+\theta_{K, e l e_{2}} \mathbf{d}_{K, e_{2}}, \\
\mathbf{d}_{K_{e}, e \ell} & =\eta_{K_{e}, e \ell e_{1}^{\prime}} \mathbf{d}_{K, e_{1}^{\prime}}+\eta_{K_{e}, e \ell e_{2}^{\prime}} \mathbf{d}_{K, e_{2}^{\prime}} .
\end{aligned}
$$

In the figure below we show this construction in the case in which the element $K$ is an interior triangle and the quadrature rule on the edges is the two-point Gauss rule. The quadrature points are indicated by a ' $\Delta$ '.

Now, if we associate with $\mathbf{d}_{K, e}$ the value

$$
\Delta_{K, e}=\left\{\begin{array}{l}
\bar{u}_{K_{e}}-\bar{u}_{K}, \text { if } e \notin \partial \Omega, \\
\bar{\gamma}_{e}-\bar{u}_{K}, \text { if } e \in \partial \Omega,
\end{array}\right.
$$

and with $-\mathbf{d}_{K, e \ell}$ and $\mathbf{d}_{K_{e}, e \ell}$ the values

$$
\begin{aligned}
-\tilde{u}_{K, e l} & =-u_{h}\left(x_{e l}^{i n t(K)}\right)+\bar{u}_{K}, \\
\tilde{u}_{K_{e}, e l} & =u_{h}\left(x_{e l}^{e x t(K)}\right)-\bar{u}_{K_{e}},
\end{aligned}
$$




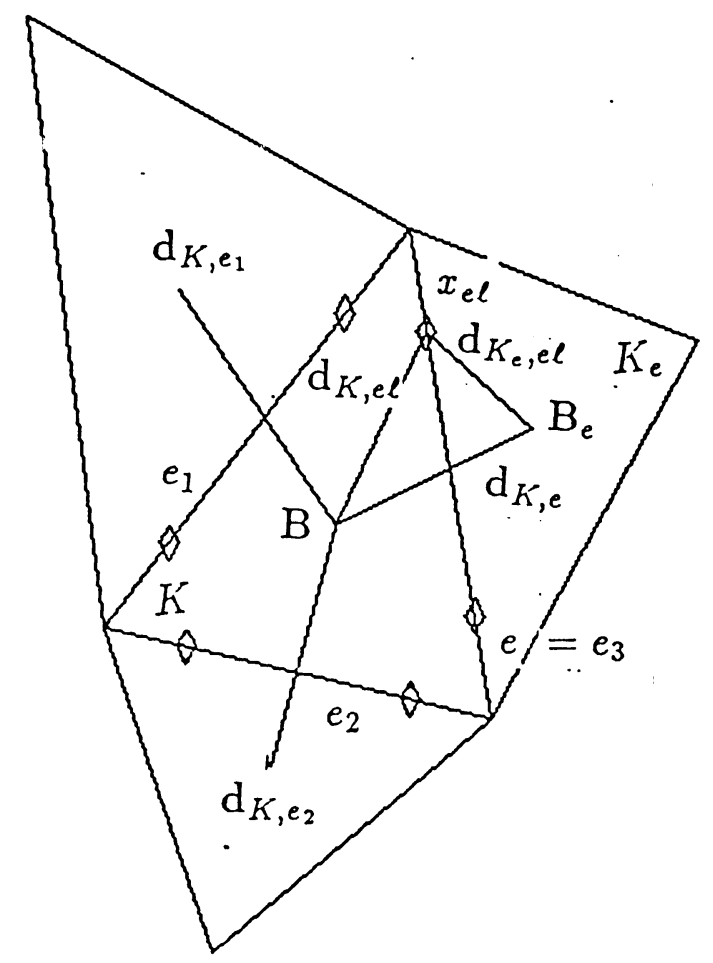

respectively, we have

$$
\begin{aligned}
-\tilde{u}_{K, e \ell} & =\left[\theta_{K, e l e_{1}} \Delta_{K, e_{1}}+\theta_{K, e l e_{2}} \Delta_{K, e_{2}}\right]+0\left(h^{2}\right), \\
\tilde{u}_{K_{e}, e \ell} & =\left[\eta_{K_{e}, e l e_{1}^{\prime}} \Delta_{K, e_{1}^{\prime}}+\eta_{K_{e}, e l e_{2}^{\prime}} \Delta_{K, e_{2}^{\prime}}\right]+0\left(h^{2}\right),
\end{aligned}
$$

in the sense of the truncation error analysis. (Compare with (2.13c).) Thus, the conditions

$$
\begin{gathered}
\tilde{u}_{K, e \ell} \in I\left(0, b \tilde{U}_{K, e \ell}^{\theta}\right), \\
\tilde{u}_{K_{e}, e \ell} \in I\left(0, b \tilde{U}_{K, e \ell}^{\eta}\right),
\end{gathered}
$$

where $b \geq 1$ and

$$
\begin{aligned}
& \tilde{U}_{K, e \ell}^{\theta}=-\left[\theta_{K, e l e_{1}} \Delta_{K, e_{1}}+\theta_{K, e \ell e_{2}} \Delta_{K, e_{2}}\right], \\
& \tilde{U}_{K, e \ell}^{\eta}=\left[\eta_{K_{e}, e \ell e_{1}^{\prime}} \Delta_{K, e_{1}^{\prime}}+\eta_{K_{e}, e \ell e_{2}^{\prime}} \Delta_{K, e_{2}^{\prime}}\right],
\end{aligned}
$$

are naturally satified (in the sense of the truncation errorr analysis) away from critical points. If $M$ is some upper bound of the second derivatives of $u$, then the conditions

$$
\tilde{u}_{K, e \ell} \in \begin{cases}{\left[I\left(0, b \tilde{U}_{K, e \ell}^{\theta}\right)\right] \cap I\left(0, b \tilde{U}_{K, e \ell}^{\eta}\right) \cup\left[-M h^{2}, M h^{2}\right],} & \text { if } e \notin \partial \Omega, \\ I\left(0, b \tilde{U}_{K, e \ell}^{\theta}\right) \cup\left[-M h^{2}, M h^{2}\right], & \text { if } e \in \partial \Omega,\end{cases}
$$


are satisfied uniformly. Moreover, these conditions (with $M=0$ ) imply conditions (2.9) provided the coefficients $\theta_{K, \ell \ell d}$ and $\eta_{K_{e}, e \ell d}$ are nonnegative. In order to guarantee this last property it is clear, from (2.13) and (2.14), that we have to restrict ourselves to consider a special class of triangulations $\mathcal{T}_{h}$ that we introduce next.

Definition 2.5. A triangulation $\mathcal{T}_{h}$ is said to be a B-triangulation if for each $\mathrm{d}_{K, \text { el }}$ and $\mathbf{d}_{K_{e}, e l}$ it is possible to pick the vectors $\mathbf{d}_{K, e_{1}}, \mathbf{d}_{K, e_{2}}, \mathbf{d}_{K, e_{1}^{\prime}}$ and $\mathbf{d}_{K, e_{2}^{\prime}}$ such that the coefficients $\theta_{K, e \ell e_{1}}, \theta_{K, e l e_{2}}, \eta_{K_{e}, e \ell e_{1}^{\prime}}$, and $\eta_{K_{e}, e \ell e_{2}^{\prime}}$ are nonnegative.

Definition 2.6. A family of triangulation $\mathcal{F}=\left\{\mathcal{T}_{h}\right\}_{h>0}$ is said to be $\mathrm{B}$-uniform if each triangulation $\mathcal{T}_{h}$ is a $\mathbf{B}$-triangulation, and there is a constant $\mu$ such that

$$
\theta_{K, e \ell d}, \eta_{K_{e}, e \ell d^{\prime}} \in[0, \mu], \quad \forall K \in \mathcal{T}_{h}, \quad \forall \mathcal{T}_{h} \in \mathcal{F} .
$$

In the next section we shall give examples of this kind of triangulations. We are now ready to define the $\Lambda \Pi_{h}$-projections. Let us denote by \#e(K) the number of edges of the element $K$. The conditions $(2.15 \mathrm{a})$ and $(2.15 \mathrm{~b})$ represent $\# e(K) \times L$ restrictions which, together with the condition

$$
\int_{K} \tilde{u}_{h}=0
$$

define a nonempty convex set $C\left(K ; \bar{u}_{h}\right) \subset V(K)$. We can now define the projection $\Lambda \Pi_{h}$ as follows:

$$
\begin{aligned}
\Lambda \Pi_{h}: V_{h} & \longmapsto V_{h} \\
u_{h} & \longmapsto w_{h}
\end{aligned}
$$

such that

$$
\left.w_{h}\right|_{K} \text { is a projection of }\left.u_{h}\right|_{K} \text { into } C\left(K ; \bar{u}_{h}\right) \text {. }
$$

Notice that we did not specify the very form of the local projection in (2.17b). We can take, for example, the $L^{2}$-projection into $C\left(K ; \bar{u}_{h}\right)$. In this case, to carry out the projection amounts to solve a minimization problem, which can be reduced to a one dimensional maximization problem via a duality argument, see [3]. In fact, thanks to (2.14c), in most elements we have $\left.u_{h}\right|_{K} \in C\left(K ; \bar{u}_{h}\right)$, and so the operator $\left.\Lambda \Pi_{h}\right|_{K}$ becomes the identity. Thus, if the exact solution is piecewise smooth it is reasonable, from the computational point of view, to have a 'very complicated' projection into $C\left(K ; \bar{u}_{h}\right)$. On the other hand, as the projection is actually carried out only very near the discontinuities, it is not necessary to define it in a very sophisticated way. Some practical implementations of this projection are considered in Section 3. 
Note also that if $K$ is a triangle and $V(K)=P^{1}(K)$, or $V(K)=P^{2}(K)$, then from the fact that $w_{h} \in C\left(K ; \bar{u}_{h}\right)$ we deduce easily that there is a constant $c_{o}$ such that:

$$
\left\|w_{h}\right\|_{L^{1}(K)} \leq c_{o}\left\{\sum_{e \in \partial K}\left|\bar{u}_{K}-\bar{u}_{K_{e}} \| e\right|\right\} h_{K},
$$

where the constant $c_{o}$ depends solely on the parameter $\sigma$ of the triangulation, see (2.6). The same property is verified if $K$ is a rectangle and $V(K)=Q^{1}(K)$. We can define $\left.\Lambda \Pi_{h}\right|_{h}$ in order to enforce (2.18a) without damaging the accuracy of the method. If we set:

$$
\begin{gathered}
Z\left(K, \bar{u}_{h}\right)=\left\{v_{h} \in V_{h}: \text { if } v_{h}\left(x_{e l}\right)=\bar{u}_{K}, \quad \forall e \in \partial K, l=1, \cdots, L\right. \\
\text { then } \left.v_{h}(x)=\bar{u}_{K}, \quad \forall x \in K\right\},
\end{gathered}
$$

and we replace $(2.17 \mathrm{~b})$ by

$$
\left.w_{h}\right|_{K} \text { is a projection of }\left.u_{h}\right|_{K} \text { into } C\left(K ; \bar{u}_{h}\right) \cap Z\left(K ; \bar{u}_{h}\right) \text {, }
$$

then property (2.18a) is always veryfied.

Thus, we have proven the following result.

Proposition 2.7. Let $\Lambda \Pi_{h}$ be the projection defined by (2.17)and Let $\mathcal{F}$ be a $\mathbf{B}$ uniform family of triangulations. Set $w_{h}=u_{h}+\delta \cdot \Delta t^{m} L_{h}\left(u_{h}, \gamma_{h}\right)$, assume that $u_{h}=$ $\Lambda \Pi_{h}\left(u_{h}\right)$, and suppose that

$$
\bar{u}_{h}, \gamma_{h} \in[a, b] .
$$

Then

$$
\bar{w}_{h} \in\left[a-M h^{2}, b+M h^{2}\right],
$$

provided

$$
c f l \leq \frac{1}{\delta(1+4 b \mu) \max \{\# e(K)\}} .
$$

Moreover,

$$
\left\|w_{h}\right\|_{L^{1}(\Omega)} \leq c_{o}\left\|\bar{w}_{h}\right\|_{B V(\Omega)} h
$$

Proof. Property (2.18b) is a simple consequence of (2.18a) and of the definition of the total variation of $\bar{u}_{h}$ :

$$
\left\|\bar{u}_{h}\right\|_{B V(\Omega)}=\sum_{K \in \mathcal{T}_{h}}\left\{\sum_{e \in \partial K \backslash \partial \Omega}\left|\bar{u}_{K}-\bar{u}_{K_{e}}\right||e|+\sum_{e \in \partial K \cap \partial \Omega}\left|\bar{u}_{h}-\bar{\gamma}_{h}\right|\right\},
$$


Now, we only have to prove condition (2.19). This condition is nothing but another version of condition (2.11)-(2.10). Consider the following expression:

$$
\Psi=\sum_{e \in \partial K \backslash \partial \Omega} \Theta_{e}+\sum_{e \in \partial K \cap \partial \Omega} \sum_{l=1}^{L} \Theta_{e l} .
$$

By the definition of $\Theta_{e}$ and $\Theta_{e l},(2.10)$, we can write

$$
\begin{aligned}
\Psi= & \sum_{e \in \partial K \backslash \partial \Omega} \sum_{\ell=1}^{L} \frac{\omega_{\ell}|e|}{|K|}\left[-h_{e l, 2}\right] \\
& +\sum_{e \in \partial K \backslash \partial \Omega} \sum_{d \in \partial K} \sum_{\ell=1}^{L} \frac{\omega_{\ell}|d|}{|K|}\left[h_{d \ell, 1}\right] \theta_{K, d \ell e} \\
& +\sum_{e \in \partial K \backslash \partial \Omega} \sum_{d \in \partial K \backslash \partial \Omega} \sum_{\ell=1}^{L} \frac{\omega_{\ell}|d|}{|K|}\left[h_{d \ell, 2}\right] \eta_{K_{d}, d \ell e} \\
& +\sum_{e \in \partial K \cap \partial \Omega} \sum_{l=1}^{L} \frac{\omega_{l}|e|}{|K|}\left[-h_{e l, 2}\right] \\
& +\sum_{e \in \partial K \cap \partial \Omega} \sum_{l=1}^{L} \omega_{l} \sum_{d \in \partial K} \sum_{\ell=1}^{L} \frac{\omega_{\ell}|d|}{|K|}\left[h_{d \ell, 1}\right] \theta_{K, d \ell e} \\
& +\sum_{e \in \partial K \cap \partial \Omega} \sum_{l=1}^{L} \omega_{l} \sum_{d \in \partial K \backslash \partial \Omega} \sum_{\ell=1}^{L} \frac{\omega_{\ell}|d|}{|K|}\left[h_{d \ell, 2}\right] \eta_{K_{d}, d \ell e} .
\end{aligned}
$$

Thus,

$$
\begin{aligned}
\Delta t^{m} \Psi & \leq c f l \sum_{e \in \partial K \backslash \partial \Omega} 1+c f l \sum_{e \in \partial K} \sum_{d \in \partial K} \sum_{\ell=1}^{L} \omega_{\ell} \theta_{K, d \ell e}+c f l \sum_{e \in \partial K} \sum_{d \in \partial K \backslash \partial \Omega} \sum_{\ell=1}^{L} \omega_{\ell} \eta_{K_{d}, d \ell e} \\
& \leq c f l\left[\# e(K)+\sum_{d \in \partial K} \sum_{\ell=1}^{L} \omega_{\ell} \sum_{e \in \partial K} \theta_{K, d \ell e}+\sum_{d \in \partial K \backslash \partial \Omega} \sum_{\ell=1}^{L} \omega_{\ell} \sum_{e \in \partial K} \eta_{K_{d}, d \ell e}\right] \\
& \leq c f l\left[\# e(K)+\sum_{d \in \partial K} \sum_{\ell=1}^{L} \omega_{\ell} 4 b \mu\right] \\
& \leq c f l \# e(K)[1+4 b \mu] .
\end{aligned}
$$

This proves the result. $\square$ 
In this way, we have obtained a class of local projections $\Lambda \Pi_{h}$ which enforce maximum principles on the RKDG-methods. In particular, if we set $M=0$, Proposition 2.7 and Lemma 2.3 guarantee that $u_{h} \in\left[a_{0}, b_{0}\right]$ provided $u_{0}, \gamma_{h} \in\left[a_{0}, b_{0}\right]$. Notice, however, that there are some values of the boundary condition $\gamma_{h}$ which are irrelevant and should not be taken into consideration when carrying out the projection $\Lambda \Pi_{h}$, for they could destroy the accuracy of the methods. Those values are the so-called outflow values, i.e., the values $\gamma_{e l}$ such that $\mathbf{f}^{\prime}\left(\gamma_{e l}\right) \cdot \mathbf{n}_{e, K}>0$. Thus, to avoid this inconvenience, we simply replace $\gamma_{e l}$ by

$$
\gamma_{e l}^{\prime}= \begin{cases}\gamma_{e l}, & \text { if } \mathbf{f}^{\prime}\left(\gamma_{e l}\right) \cdot \mathbf{n}_{e, K} \leq 0, \\ \tilde{U}_{e l}^{\theta}, & \text { otherwise }\end{cases}
$$

in (2.9). This completes our boundary conditions treatment. Proposition 2.7 remains valid in this case.

2.5. On $\mathbf{B}$ triangulations. We now give two main examples of $\mathbf{B}$-uniform families of triangulations. We begin with a very simple result which shows that a B-uniform fammily of triangulations need not to be regular.

Proposition 2.8. Let $\mathcal{F}$ be a family of triangulations made of rectangles. Then $\mathcal{F}$ is a B-uniform family. Moreover, $\mu=1$.

A more delicate case is the following.

Proposition 2.9. Let $\mathcal{F}$ be a regular family of triangulations made of acute triangles. Then $\mathcal{F}$ is a B-uniform family. Moreover, $\mu=2 \sigma^{3}\left(1+\sigma^{2}\right)^{3 / 2}$.

Proof. First, let us prove that each triangulation $\mathcal{T}_{h} \in \mathcal{F}$ is a $\mathbf{B}$-triangulation. Consider the figure below. The straight line $\ell$ is parallel to $\mathbf{a}_{2}-\mathbf{a}_{1}$, the line $\ell_{1}$ is parallel to $\mathbf{b}_{1}-\mathbf{a}_{2}$, and the line $\ell_{2}$ to $\mathbf{b}_{3}-\mathbf{a}_{1}$. The point $\mathbf{P}_{i}$ is the intersection of $\ell$ with $\ell_{i}, i=1,2$. The triangle whose barycenter is $\mathbf{B}$ (resp., $\mathbf{B}_{i}$ ) will be denoted by $T$ (resp., $T_{i}$ ). We shall prove that the angle $\left(\alpha_{1}+\alpha_{2}\right)$ is bounded below by a positive constant depending solely on $\sigma$. This implies that $\mathcal{T}_{h}$ is a B-triangulation.

Note that

$$
\left|\mathbf{B}_{1}-\mathbf{B}\right| \sin \left(\alpha_{1}\right)=\left|\mathbf{B}_{1}-\mathbf{P}_{1}\right| \sin \psi_{1} \text {. }
$$

By construction $\mathbf{B}_{1}-\mathbf{B}=\frac{1}{3}\left(\mathbf{b}_{1}-\mathbf{a}_{1}\right)=\frac{1}{3}\left(\mathbf{b}_{1}-\mathbf{a}_{2}\right)+\frac{1}{3}\left(\mathbf{a}_{2}-\mathbf{a}_{1}\right)$, and $\mathbf{B}_{1}-\mathbf{P}_{1}=\frac{1}{3}\left(\mathbf{b}_{1}-\mathbf{a}_{2}\right)$. Thus:

$$
-\sin \left(\alpha_{1}\right) \geq \frac{\left|\mathbf{b}_{1}-\mathbf{a}_{2}\right|}{\left|\mathbf{b}_{1}-\mathbf{a}_{2}\right|+\left|\mathbf{a}_{2}-\mathbf{a}_{1}\right|} \sin \left(\psi_{1}\right) .
$$

By the regularity condition (2.6) we have:

$$
\begin{aligned}
& \frac{1}{\sigma} \leq \frac{\left|\mathbf{a}_{2}-\mathbf{a}_{1}\right|}{\left|\mathbf{a}_{2}-\mathbf{a}_{3}\right|} \leq \sigma, \\
& \frac{1}{\sigma} \leq \frac{\left|\mathbf{a}_{2}-\mathbf{a}_{3}\right|}{\left|\mathbf{b}_{1}-\mathbf{a}_{2}\right|} \leq \sigma,
\end{aligned}
$$




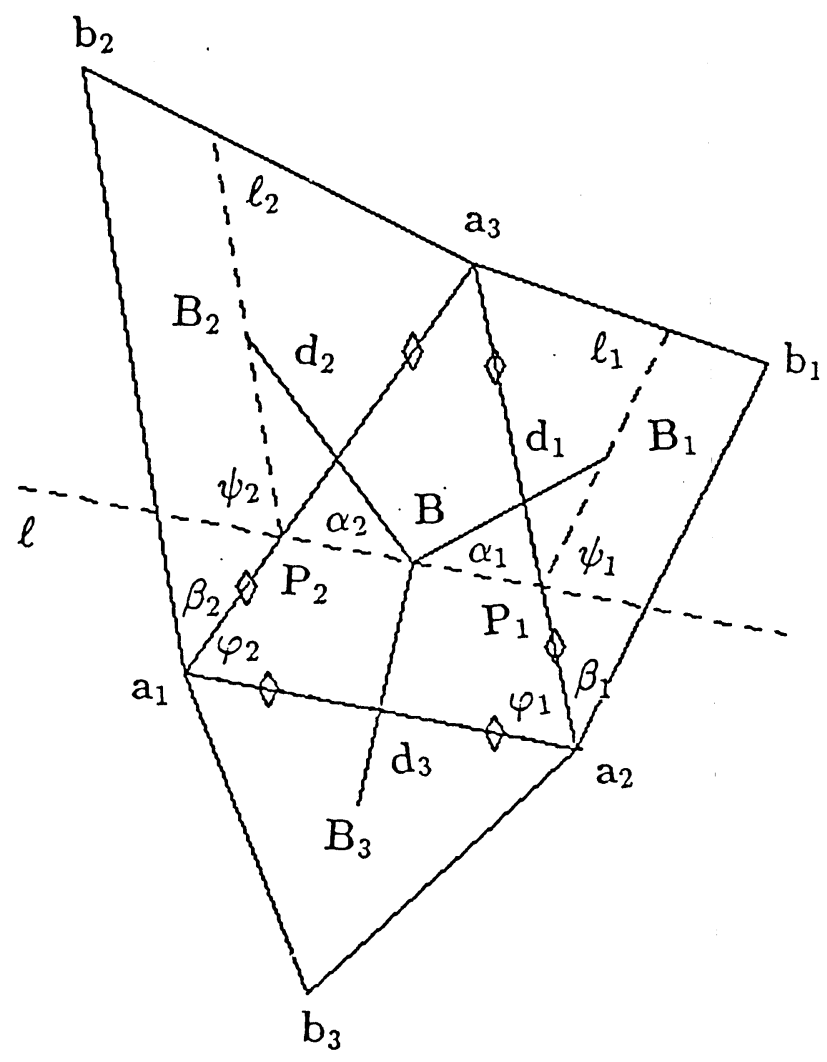

and so

$$
\sin \left(\alpha_{1}\right) \geq \frac{1}{1+\sigma^{2}} \sin \left(\psi_{1}\right)
$$

If we set $\operatorname{cotn}\left(\alpha_{0}\right)=\sigma$ the regularity condition (2.6) can be restated as follows: every angle of $K$ is bigger than $2 \alpha_{0}$. This is the so-called Zlámal condition, see [4]. Thus,

$$
\begin{aligned}
& \psi_{1}=\pi-\varphi_{1}-\beta_{1} \in\left[\frac{\pi}{2}-\varphi_{1}, \pi-2 \alpha_{0}-\varphi_{1}\right], \\
& \psi_{2}=\pi-\varphi_{2}-\beta_{2} \in\left[\frac{\pi}{2}-\varphi_{2}, \pi-2 \alpha_{o}-\varphi_{2}\right] .
\end{aligned}
$$

If $\psi_{1} \geq \frac{\pi}{2}$, then $\sin \left(\psi_{1}\right) \geq \sin 4 \alpha_{0}$. If $\psi_{1} \in\left[\frac{\pi}{2}-\varphi_{1}, \frac{\pi}{2}\right]$, then $\sin \left(\psi_{1}\right) \geq \sin \left(\frac{\pi}{2}-\varphi_{1}\right)$. If $\psi_{1}$ and $\psi_{2}$ are smaller than $\frac{\pi}{2}$,

$$
\begin{aligned}
\max \left\{\sin \left(\psi_{1}\right), \sin \left(\psi_{2}\right)\right\} & \geq \max \left\{\sin \left(\frac{\pi}{2}-\varphi_{1}\right), \sin \left(\frac{\pi}{2}-\varphi_{2}\right)\right\} \\
& \geq \inf _{\varphi_{1}, \varphi_{2}} \max \left\{\sin \left(\frac{\pi}{2}-\varphi_{1}\right), \sin \left(\frac{\pi}{2}-\varphi_{2}\right)\right\} .
\end{aligned}
$$

By summetry, the infimum is attained when $\varphi_{1}=\varphi_{2}=\varphi$, so that $\frac{\pi}{2}-\varphi=\frac{\varphi_{3}}{2} \geq \alpha_{0}$, and so $\max \left\{\sin \left(\psi_{1}\right), \sin \left(\psi_{2}\right)\right\} \geq \sin \alpha_{0}$.

As a consequence

$$
\max \left\{\sin \left(\alpha_{1}\right), \sin \left(\alpha_{2}\right)\right\} \geq \sin ^{3} \alpha_{0}=\left(1+\sigma^{2}\right)^{-3 / 2},
$$


which implies that $\left(\alpha_{1}+\alpha_{2}\right)$ is uniformly bounded from below by a strictly positive constant depending solely on $\sigma$. This shows that any vector can be written either as negative combinations of the vectors $\mathrm{d}_{1}, \mathrm{~d}_{2}$, and $\mathrm{d}_{3}$, or as positive combinations of them. This implies that $\mathcal{F}$ is a family of $\mathrm{B}$-triangulations.

Now let us prove the estimate of $\mu$. Set $\mathbf{d}=\nu_{1} \mathbf{d}_{1}+\nu_{2} \mathbf{d}_{2}$, where the coefficients $\nu_{i}$ are nonnegative. Let $\xi_{12}$ be the angle between $\mathrm{d}_{1}$ and $\mathrm{d}_{2}$, and let $\xi$ be the one between $\mathrm{d}$ and $\mathrm{d}_{2}$. Let us estimate $\nu_{1}$. By definition.

$$
\begin{aligned}
\nu_{1} & =-\frac{d \cdot d_{2}^{\perp}}{d_{1} \cdot d_{2}^{\perp}} \\
& \leq \frac{|d|}{\left|d_{1}\right|} \cdot \frac{|\sin \xi|}{\left|\sin \xi_{12}\right|} .
\end{aligned}
$$

If $\xi_{12} \in[0, \pi / 2]$, then $\xi \in\left[0, \xi_{12}\right]$, and so $\frac{|\sin \xi|}{\left|\sin \xi_{12}\right|} \leq 1$. If $\xi_{12} \in[\pi / 2, \pi]$, then $\alpha_{1}+\alpha_{2}=\pi-\xi_{12} \in[0, \pi / 2)$ and so,

$$
\sin \xi_{12} \geq \max \left\{\sin \left(\alpha_{1}\right), \sin \left(\alpha_{2}\right)\right\} \geq \sin ^{3} \alpha_{0} .
$$

Thus,

$$
\nu_{1} \leq \frac{|\mathbf{d}|}{\left|\mathbf{d}_{1}\right|} \cdot \sin ^{-3} \alpha_{0} .
$$

But, by construction, $\left|\mathbf{d}_{1}\right|=\frac{1}{3}\left|\mathbf{b}_{1}-\mathbf{a}_{1}\right|$. If the edge $\mathbf{a}_{2} \mathbf{a}_{3}$ lies on $\partial \Omega$ then, $b_{1}$ is the middle point of it, by construction. This implies that $\left|\mathbf{d}_{1}\right| \geq \rho_{T} / 3$. If the edge $\mathbf{a}_{2} \mathbf{a}_{3}$ does not lie on $\partial \Omega$, a simple calculation shows that $\left|\mathbf{d}_{1}\right| \geq\left(1+\frac{1}{\sigma^{2}}\right) \rho_{T} / 3$. The regularity of the triangulation has strongly been used in this last step. Thus, we always have that $\left|\mathrm{d}_{1}\right| \geq \rho_{T} / 3$.

It remains to estimate $|\mathrm{d}|$. The cases of interest are when (i) $\mathrm{B}+\mathrm{d}$ lies in the border of the triangle $T$, and when $\mathbf{B}_{i}+\mathbf{d}$ lies in the border of the triangle $T$, for $i=1,2,3$. The first case corresponds to the equation (2.13c), whereas the second to equation (2.13d). In the first case we simply have $|\mathbf{d}| \leq 2 h_{T} / 3$. In the second we obtain $|\mathbf{d}| \leq 2 h_{T_{i}} / 3 \leq 2 \sigma^{2} h_{T} / 3$, as a consequence of the regularity of the triangulation. As $\sigma \geq \sqrt{3}$ we have $|\mathrm{d}| \leq 2 \sigma^{2} h_{T} / 3$ in all the cases under consideration. Thus,

$$
\nu_{1} \leq \frac{2 \sigma^{2} h_{T} / 3}{\rho_{T} / 3} \sin ^{-3}\left(\alpha_{0}\right) \leq 2 \sigma^{3}\left(1+\sigma^{2}\right)^{3 / 2}
$$

This proves the result.

2.6. Stability and Convergence. We summarize the results obtained above in the following theorem. 
THEOREM 2.10. Consider the RKDG method (2.1), where the operator $L_{h}$ is defined by (2.5)-(2.4)-(2.3)-(2.2), and the projection $\Lambda \Pi_{h}$ is defined by (2.17)-(2.15-(2.20). Assume that the family of triangulations $\mathcal{F}$ is regular and $\mathbf{B}$-uniform. Suppose that $V(K) \supset$ $P^{k}(K), \quad \forall K \in \mathcal{T}_{h}, \quad \forall \mathcal{T}_{h} \in \mathcal{F}$, and that the quadrature rule over the edges is exact for polynomials of degree $(2 k+1)$, and that the quadrature rule over the elements is exact for polynomials of degree $2 k$. Then:

(1) The RKDG method is formally uniformly $k+1$-order accurate in time and space if $\Delta t=O(h)$;

(2) The approximate solution generated by the RKDG method verifies the maximum principle (2.8b) if the $c f l$-condition (2.19) is verified with $\delta=\max _{i, l}\left|\frac{\beta_{i, l}}{\alpha_{i, l}}\right|$;

(3) The approximate solution converges to a weak solution of (1.1) if there is a constant $C$ such that $\left\|\bar{u}_{h}\right\|_{B V(\Omega)} \leq C$.

The proof of (3) is similar to the proof of the same result for the one dimensional case and will be omitted; see [7].

3. Numerical results. In this section we display some preliminary numerical results. Extensive computations, in which we explore numerically several fluxes, triangulations, finite elements, quadrature rules, and local projections, constitute the object of a forthcoming paper.

We consider triangulations made only of triangles (see Fig. 1), and we take the local finite element space $V(T)$ to be $P^{k=1}(T)$, i.e., the space of linear functions on $T$. Proposition 2.1 affirms that we can reach a second order accurate space approximation (which is in fact the best possible order of accuracy that can be reached with the given elements), provided we take a quadrature rule for the edges exact for polynomials of degree 3 , and a quadrature rule for the elements exact for polynomials of $P^{2}$. Accordingly, we take the two-points Gauss quadrature rule for the edges, and the three mid-points rule for the triangles. We take the Godunov flux as the flux $h_{e, T}$, and the Runge-Kutta time discretization parameters of order two; see Table 1 . To complete the definition of this RKDG ${ }^{1}$ method (which is formally uniformly second order accurate) we need to specify the local projection, $\Lambda \Pi_{h}$.

This projection is defined as described in section 2.4, only, the points $x_{e \ell}$ associated to the quadrature rule of the edges are replaced by the points associated to the degrees of freedom, the midpoints of the edges. In this way, four conditions are to be enforced by the projection on each triangle. Each of the degrees of freedom generates a single condition $(2.15 \mathrm{~b})$. The fourth is provided by the conservativity condition (2.15c). First, the projection $\Lambda \Pi_{h}$ enforces each of the conditions (2.15b) independently of each other. This constitutes three simple one-dimensional projections. After this step, the conservativity condition $(2.15 \mathrm{c})$ is enforced via a trivial arithmetic computation which leaves the conditions $(2.15 \mathrm{~b})$ verified. 
It is important to stress the fact that the choice of the degrees of freedom as the values at the midpoints of the edges of each triangle, increases the computational efficiency of the method. It allows to save time in the evaluation of the integral over the triangles, and it allows to define a simple and efficient local projection $\Lambda \Pi_{h}$.

We are going to test the RKDG ${ }^{1}$ method described above in three examples. We point out that we compute the $L^{\infty}$-error on the triangle $T$ by evaluating the error at the barycenter. The $L^{1}(T)$-error is obtained by multiplying that value by the area of the triangle. The errors are evaluated over the whole domain, unless otherwise stated. The $L^{1}$-error is divided by the area of the domain over which it has been computed. We also have to do a couple of comments about the graphic outputs. A given function $v$, which is typically either the exact solution, or its finite element approximation, is represented graphically as a surface (and its level curves). To obtain such a surface we evaluate the function $u$ at each of the points of a $70 \times 70$ uniform grid. Then, we interpolate them linearly. Finally, in the figures in which we display cuts along the diagonal od the domain $\Omega$, the solid line always represents the exact solution. The ' $+{ }^{\prime}$ represent the approximate solution. A single ' + ' per triangle has been displayed.

Example 1. In this problem, we test the capability of the method to achieve uniform second order accuracy away from discontinuities. We consider the two dimensional version of Burger's equation with periodic boundary conditions:

$$
\begin{aligned}
& \partial_{t} u+\partial_{x}\left(u^{2} / 2\right)+\partial_{y}\left(u^{2} / 2\right)=0, \quad \text { in }(0, T) \times \Omega, \\
& u(t=0, x, y)=\frac{1}{4}+\frac{1}{2} \sin (\pi(x+y)), \quad(x, y) \in \Omega,
\end{aligned}
$$

where the domain $\Omega$ is the square $[-1,1] \times[-1,1]$. We take $M=20$, and $b=3$. The triangulations $\mathcal{T}_{h, \text { unif }}$ are like the one displayed on Fig. 1 (top). (Notice that in that figure $h=1 / 16$.)

At $T=0.1$ the exact solution is smooth, see Fig. 2. On Table 2, the $L^{1}$ and $L^{\infty}$ errors at $T=0.1$ are displayed. The approximate solution (associated to the triangulation of Fig. 1 (top)) is shown on Fig. 3 and Fig. 6 (top). Uniform second order accuracy has been achieved.

At $T=0.45$ the exact solution presents a discontinuity curve, see Fig. 4. The errors away from the discontinuity curve are shown on Table 3 . The approximate solution is displayed on Fig. 5 and Fig 6 (bottom). Again, uniform second order accuracy has been achieved away from discontinuities. Notice how the discontinuity curve has been captured whithin a single element, see Fig. 6 (bottom).

Example 2. In this problem, we test the boundary treatment of the method. We consider the preceding equations, but this time we impose a boundary condition:

$$
\begin{array}{lc}
\partial_{t} u+\partial_{x}\left(u^{2} / 2\right)+\partial_{y}\left(u^{2} / 2\right)=0, & \text { in }(0, T) \times \Omega, \\
u(t=0, x, y)=\frac{1}{4}+\frac{1}{2} \sin (\pi(x+y)), & (x, y) \in \Omega, \\
u(t, x, y)=v(t, x, y)), & (x, y) \in \partial \Omega,
\end{array}
$$


where $v$ is the exact solution of problem (5.1). See [1] for a suitable interpretation of the boundary conditions. The exact solution of this problem coincides with the one of problem (5.1). Notice that since we use a pure upwind monotone flux, the outflow boundary condition, even if provided redundantly, is never used in the computation. All the discretization parameters are the same as in the preceding example.

The results are indistiguishable from the ones of the preceding problem. The $L^{\infty}$-errors are the same of those displayed on Tables 2, and 3. Thus, the boundary treatment does not introduce any spurious oscillation, maintains the maximum principle, and does not destroy the uniform accuracy of the method.

Example 3. In this last example we test the convergence of the method in the case of nonconvex fluxes. We also test the hability of the method to take advantage of nonuniform triangulations. Consider the initial boundary problem:

$$
\begin{aligned}
& \partial_{t} u+\partial_{x} f(u)+\partial_{y} f(u)=0, \\
& u(t=0, x, y)=\left\{\begin{array}{rl}
7 / 8, \text { for } x>0 \text { and } y>0, & \text { in }(0, T) \times \Omega, \\
0, \text { for } x<0 \text { and } y>0, & \text { for } x<0 \text { and } y<0, \\
1 / 4, \text { for } x>0 \text { and } y<0, & (x, y) \in \Omega,
\end{array} \quad(x, y) \in \partial \Omega,\right.
\end{aligned}
$$

where $f(u)=5\left(1 / 8+(u-1 / 2)^{3}\right), \Omega$ is the square $[-1,1] \times[-1,1]$, and $v$ is the exact solution of the corresponding Riemann problem. The exact solution has been computed following [29], and is displayed on Fig. 7. We take $M=0$ and $b=3$. On Table 4 we can see that convergence to the entropy solution is achieved.

On Fig. 8, 9, and 10 we display the approximate solutions. The approximate solution of Fig. 8 is defined on a triangulation whose triangles do not match the discontinuity curve. Nevertheless, the curve has been captured within two triangles, except at its cusp. The approximate solution of Fig. 9 has, on the contrary, been defined on a triangulation designed to fit the discontinuity curve, and to better resolve the structure of the cusp. An excellent capture of discontinuities can be observed. See also Fig. 10. On Table 5 we compare the $L^{1}$ errors of the approximate solutions under consideration.

In conclusion, the numerical results show that (i) the RKDG ${ }^{1}$ method is uniformly second order accurate away from discontinuities, that (ii) it does take advantage of suitable (nonuniform) triangulations, that (iii) it can resolve well complicated structures of the discontinuity curves, and that (iv) it converges to the entropy solution even when the fluxes are nonconvex.

4. Concluding Remarks. This paper is the fourth of a series, [5], [6], [7], in which we introduce, analyze, and test a new class of methods for numerically solving nonlinear 
hyperbolic conservation laws. These methods are called the Runge-Kutta Discontinuous Galerkin Methods. In the previous papers the one dimensional case, $d=1$, has been considered. In this paper we consider the multidimensional scalar case. A general theory for these schemes has been developed. These methods can easily handle complicated geometries, for they can be defined using quite arbitrary triangulations. For the so-called uniform triangulations, these methods are formally uniformly $(k+1)$-th order accurate (when $\Delta t=O(\Delta x))$. They can easily handle the boundary conditions. They also verify a suitable maximum principle for general nonlinearities, if the triangulations are $\mathbf{B}$-triangulations, a concept introduced in this paper. The methods are easy to code, and show high order uniform accuracy, good capture of discontinuity curves, and convergence to the entropy solution even for nonconvex nonlinearities. Extensive computational experiments for the scalar case, as well as extensions to two dimensional systems constitute the subject of ongoing work.

\section{REFERENCES.}

[1] C. Bardos, A.Y. LeRoux and J.C. Nedelec, First order quasilinear equations with boundary conditions, Comm. in P.D.E., 4 (1979), pp. 1017-1034.

[2] J.B. Bell, C.N. Dawson and G.R. Shubin, An unsplit, higher-order Godunov method for scalar conservation laws in multiple dimensions, Preprint, April 1986.

[3] G. Chavent, B. Cockburn, G. Cohen and J. Jaffré, A discontinuous finite element method for nonlinear hyperbolic equations, Innovative Numerical Methods in Engineering, Proceedings of the 4th. International Symposium, Georgia Institute of Technology, Atlanta, Georgia, Springer Verlag, 1986, pp. 337-342.

[4] P.G. Ciarlet, The Finite Element Method for Elliptic Problems, North Holland, 1975.

[5] B. Cockburn and C.-W. Shu, The Runge-Kutta local projection $P^{1}$ Discontinuous Galerkin finite element method for scalar conservation laws, Institute for Mathematics and its Applications Preprint Series \# 388, U. of Minnesota, (1988); Proceedings of First National Fluid Dynamics Congress, Cincinnati, Ohio, July 24-28, 1988.

[6] B. Cockburn and C.-W. Shu, TVB Runge-Kutta local projection discontinuous-Galerkin finite element method for conservation laws II: General framework, Institute for Mathematics and its Applications Preprint Series \# 392, U. of Minnesota (to appear in Math. Comp.).

[7] B. Cockburn, S.-Y. Lin and C.-W. Shu, TVB Runge-Kutta local projection discontinuous-Galerkin finite element method for conservation laws III: One Dimensional Systems, Institute for Mathematics and its Applications Preprint Series \# 415, U. of Minnesota (to appear in J. Comput. Phys.).

[8] B. CockBurn, The quasi-monotone schemes for scalar conservation laws, I, Institute for Mathematics and its Applications Preprint Series \# 263, University of Minnesota, (1986) (to appear in SIAM J. Numer. Anal.). 
[9] , The quasi-monotone schemes for scalar conservation laws, II, Institute for Mathematics and its Applications Preprint Series \# 268, University of Minnesota, (1986) (to appear in SIAM J. Numer. Anal.).

[10] - The quasi-monotone schemes for scalar conservation laws, III, Institute for Mathematics and its Applications Preprint Series \# 277, University of Minnesota, (1986) (to appear in SIAM J. Numer. Anal.).

[11] M. Crandall and A. Majda, Monotone difference approximations for scalar conservation laws, Math of Comp., 34 (1980), pp. 1-21.

[12] E. Giusti, Minimal surfaces and functions of bounded variation, Birkhäuser, 1984.

[13] J. Goodman and R. LeVeque, On the accuracy of stable schemes for $2 D$ scalar conservation laws, Math. Comp., 45 (1985), pp. 15-21.

[14] A. HARTEN, On a class of high-resolution total-variation-stable finite-difference schemes, SIAM J. Numer. Anal., 21 (1984), pp. 1-23.

[15] , Preliminary results on the extension of ENO schemes to two-dimensional problems, in Proceedings of the International Conference on Hyperbolic Problems, Saint-Etienne, January 1986.

[16] - ENO Schemes with Subcell Resolutions, ICASE Report 87-56, NASA Langley Research Center, August 1987. To appear in J. Comp. Phys.

[17] A. HARTEN AND S. Osher, Uniformly high order accurate non-oscillatory schemes, I, SIAM J. Numer. Anal., 24 (1987), pp. 279-309.

[18] A. Harten, B. Engquist, S. Osher and S. Chakravarthy, Uniformly high order accurate non-oscillatory schemes, III, J. Comput. Phys., 71 (1987), pp. 231-303.

[19] T. Hughes And A. Brook, Streamline Upwind-Petrov-Galerkin formulations for Convection dominated flows with particular emphasis on the incompressible Navier-Stokes equations, Comp. Meth. in App. Mech. and Eng., 32 (1982), pp. pp. 199-259.

[20] T. Hughes and M. Mallet, A High-Precision Finite Element Method for Shock-Tube Calculations, Finite Element in Fluids, $\underline{6}, 339$ (1985).

[21] T. Hughes, L.P. Franca, M. Mallet and A. Misukami, A new finite element formulation for computational fluid dynamics, I, Comp. Meth. in App. Mech. and Eng., 54 (1986), pp. 223-234.

[22] T. Hughes, L.P. Franca, M. Mallet and A. Misukami, A new finite element formulation for computational fluid dynamics, II, Comp. Meth. in App. Mech. and Eng., 54 (1986), pp. 341-355.

[23] T. Hughes, L.P. Franca, M. Mallet and A. Misukami, A new finite element formulation for computational fluid dynamics, III, Comp. Meth. in App. Mech. and Eng., 58 (1986), pp. 305-328. 
[24] T. Hughes, L.P. Franca, M. Mallet and A. Misukami, $A$ new finite element formulation for computational fluid dynamics, IV, Comp. Meth. in App. Mech. and Eng., 58 (1986), pp. 329-336.

[25] C. Johnson and J. Saranen, Streamline diffusion methods for problems in fluid mechanics, Math. Comp., 47 (1986), pp. 1-18.

[26] C. Johnson And A. Szepessy, On the convergence of a finite element method for a non-linear hyperbolic conservation law, Math. Comp., 49 (1987), pp. 427-444.

[27] C. Johnson, A. Szepessy and P. Hansbo, On the convergence of shock capturing streamline diffusion finite element methods for hyperbolic conservation laws, preprint.

[28] W. B. Lindquist, The scalar Riemann problem in two spatial dimensions: piecewise smoothness of solutions and its breakdown, SIAM J. Math. Anal., 17 (1986), pp. 1178-1197.

[29] W. B. Lindquist, Construction of solutions for two-dimensional Riemann problems, Comp. \& Maths. with Appls., 12A (1986), pp. 615-630.

[30] B. J. LUCIER, Regularity through approximation for scalar conservation laws, IMA Preprint \# 336 (1987).

[31] B. J. Lucier, A moving mesh numerical method for hyperbolic conservation laws, Math. Comp., 46 (1986), pp. 59-69.

[32] Convergence of Generalized MUSCL Schemes, SIAM J. Numer. Anal., 22 (1984), pp. 947-961.

[33] S. Osher and S. Chakravarthy, High resolution schemes and the entropy condition, SIAM J. Numer. Anal., 21 (1984), pp. 955-984.

[34] R. SANDERS, A third order accurate variation no scheme for single non expansive difference scheme for single nonlinear conservation laws, Math. Comp., 51 (1988), pp. 535-558.

[35] C.W. SHU, TVB uniformly high order schemes for conservation laws, Math. of Comp., 49 (1987), pp. 105-121.

[36] - TVB boundary treatment for numerical solutions of conservation laws, Math. Comp., 49 (1987), pp. 123-134.

[37] - TVD time discretizations, SIAM J. Sci. Stat. Comput., 9 (1988), pp. 1073-1084.

[38] C.-W. Shu ANd S. Osher, Efficient Implementation of Essentially Non-Oscillatory Shock-Capturing Schemes, J. Comput. Phys., 77 (1988), pp. 439-471.

[39] Efficient Implementation of Essentially Non-Oscillatory Shock Capturing Schemes, II, ICASE Report 88-24, NASA Langley Research Center, April 1988. To appear in J. Comp. Phys.

[40] P. SWeby, High resolution schemes using flux limiters for hyperbolic conservation laws, SIAM J. Numer. Anal., 21 (1984), pp. 995-1011. 
[41] C. Tong AND Z. YU-XI, Two dimensional Riemann problems for a single conservation law, Institute of Mathematics, Academia Sinica, Preprint \# 20 (1985).

[42] C. Tong And C. Guiquiang, Some fundamental concepts about systems of two spatial dimensional conservation laws, Acta Mathematica Scientia, 6 (1986), pp. 463-474.

[43] D. WAGNeR, The Riemann problem in two space dimensions for a single conservation law, SIAM J. Math. Anal., 14 (1983), pp. 534-559.

Table 2

Example 1: Initial value problem (5.1), $t=0.1$.

\begin{tabular}{|c|c|c|c|c|}
\hline & \multicolumn{2}{|c|}{$L^{\infty}$} & \multicolumn{2}{c|}{$L^{1}$} \\
\hline & & & $10^{4} \cdot$ error & order \\
\hline & $10^{4} \cdot$ error & order & & \\
\hline & & & & \\
$1 / 2$ & 500.92 & - & 248.55 & - \\
$1 / 4$ & 118.20 & 2.08 & 61.27 & 2.02 \\
$1 / 8$ & 37.27 & 1.67 & 15.04 & 2.03 \\
$1 / 16$ & 8.65 & 2.11 & 3.73 & 2.01 \\
$1 / 32$ & 2.20 & 1.97 & 0.94 & 1.99 \\
& & & & \\
\hline
\end{tabular}

Table 3

Example 1: Initial value problem (5.1), $t=0.45$. The errors are computed in the region $[-0.2,0.4] \times[-0.2,0.4]$.

\begin{tabular}{|c|c|c|c|c|}
\hline & \multicolumn{2}{|c|}{$L^{\infty}$} & \multicolumn{2}{c|}{$L^{1}$} \\
\hline & & & $10^{4} \cdot$ error & order \\
\hline & $10^{4} \cdot$ error & order & & \\
\hline & & & & \\
$1 / 2$ & 63.71 & - & 23.61 & - \\
$1 / 4$ & 8.38 & 2.93 & 2.52 & 3.05 \\
$1 / 8$ & 3.92 & 1.10 & 0.72 & 1.98 \\
$1 / 16$ & 0.92 & 2.09 & 0.17 & 2.10 \\
$1 / 32$ & 0.22 & 2.07 & 0.03 & 2.39 \\
\hline
\end{tabular}


Table 5

Example 3: Initial boundary value problem (5.3), $t=1$.

\begin{tabular}{|c|c|c|}
\hline & \multicolumn{2}{|c|}{$L^{1}$} \\
\hline & $10^{1} \cdot$ error & order \\
\hline & & \\
\hline & & \\
$1 / 2$ & 7.17 & 0.60 \\
$1 / 4$ & 4.73 & 0.69 \\
$1 / 8$ & 2.92 & 0.77 \\
$1 / 16$ & 1.71 & 0.78 \\
$1 / 32$ & 0.99 & \\
\hline
\end{tabular}

\section{Table 6}

Example 3: Initial boundary value problem (5.3), $t=1$. Effect of the triangulation on the overall $L^{1}$-error.

\begin{tabular}{|c|c|c|}
\hline triangulation & number of elements & $10^{1} \cdot$ error \\
\hline & 2048 & 1.71 \\
$\mathcal{T}_{h=1 / 16, \text { unif }}$ & 2048 & 0.89 \\
$\mathcal{T}_{h, \text { nonunif }}$ & 8192 & 0.99 \\
$\mathcal{T}_{h=1 / 32, \text { unif }}$ & & \\
& & \\
\hline
\end{tabular}



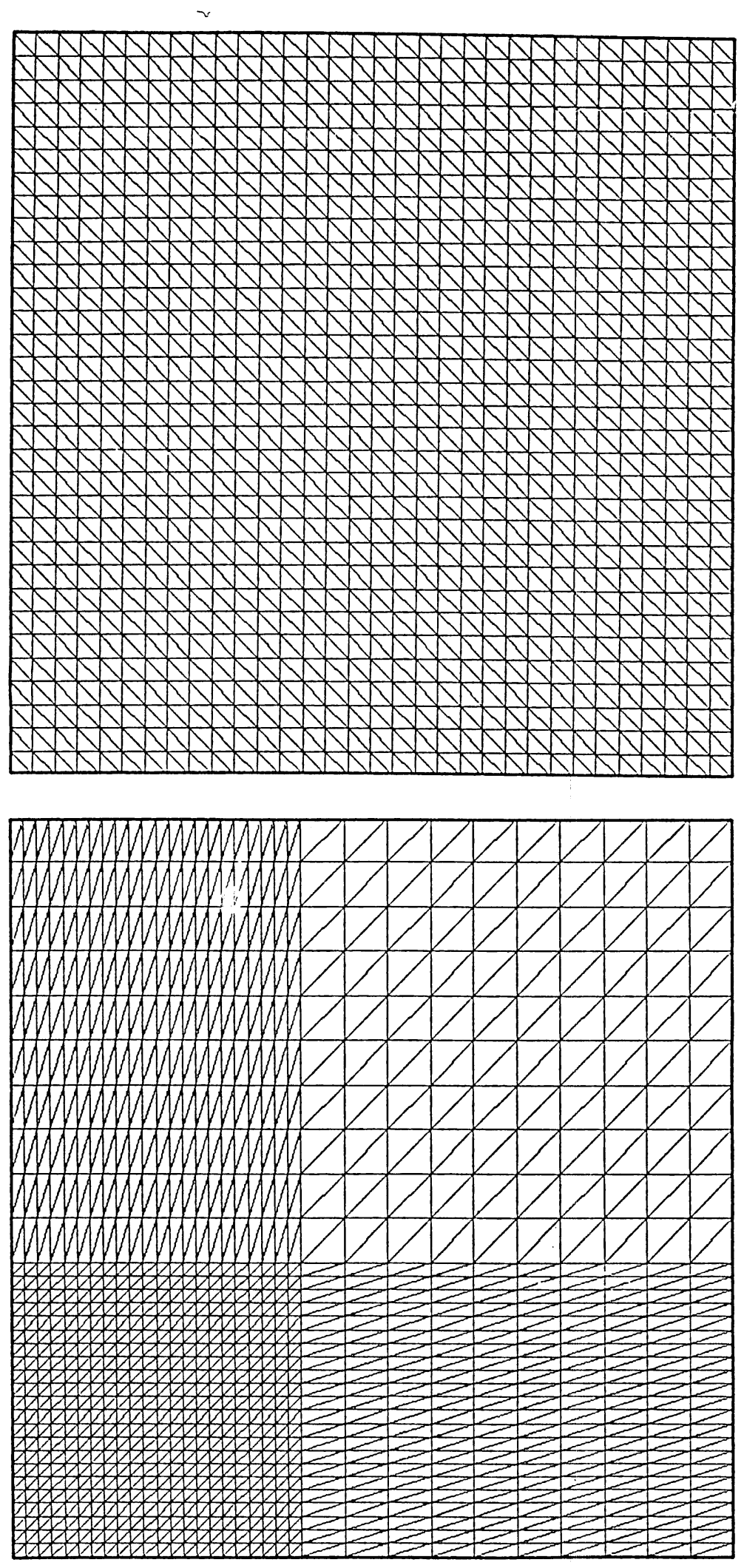

Fig. 1: The triangulations: $\mathcal{T}_{h=1 / 16, \text { unif }}$ (on top) and $\mathcal{T}_{h, \text { nonunif }}$. Both triangulations have $32 \times 32 \times 2=2048$ elements. 

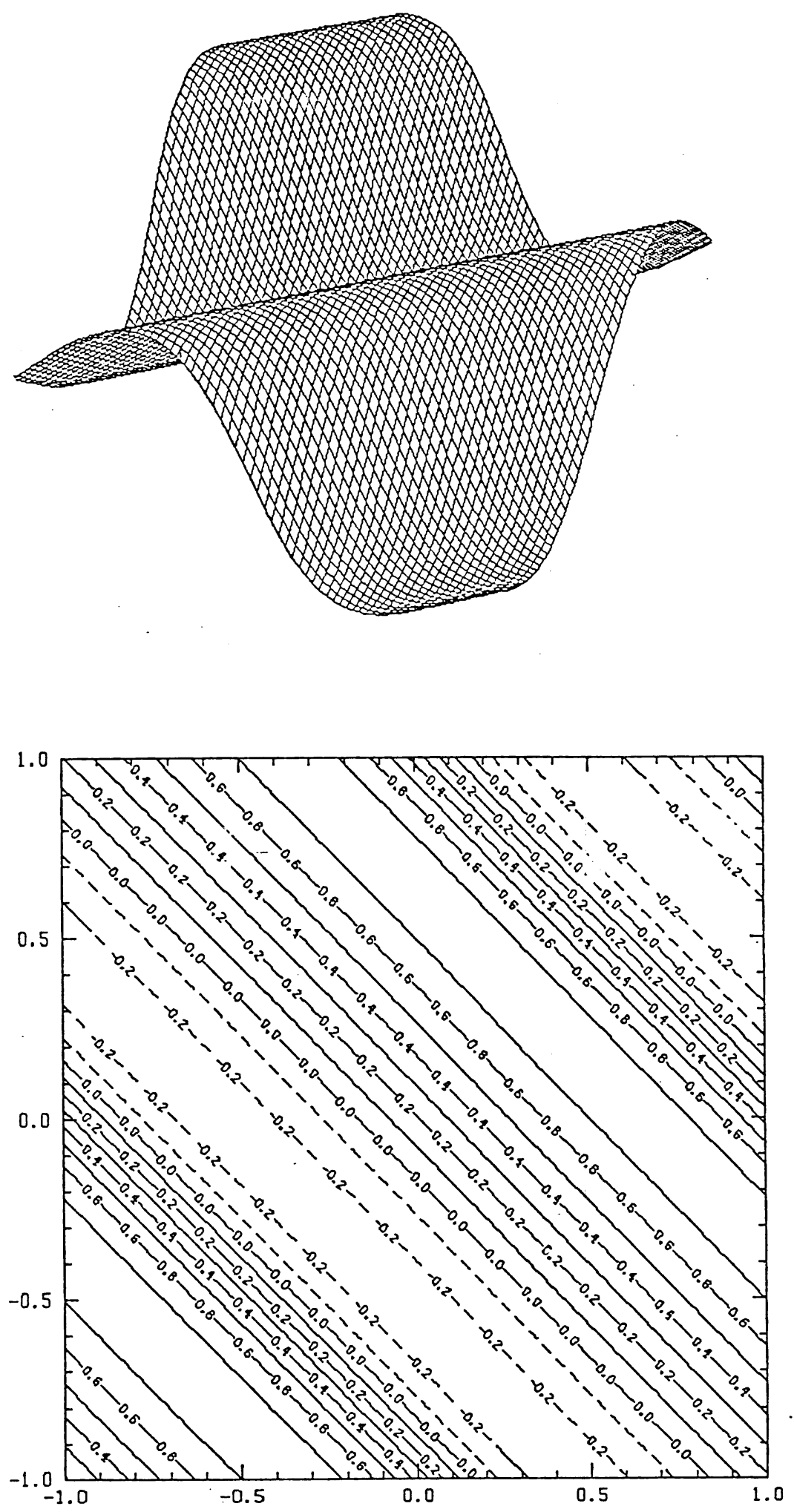

Fig. 2: The exact solution. Initial value problem (5.1), $T=0.1$. 

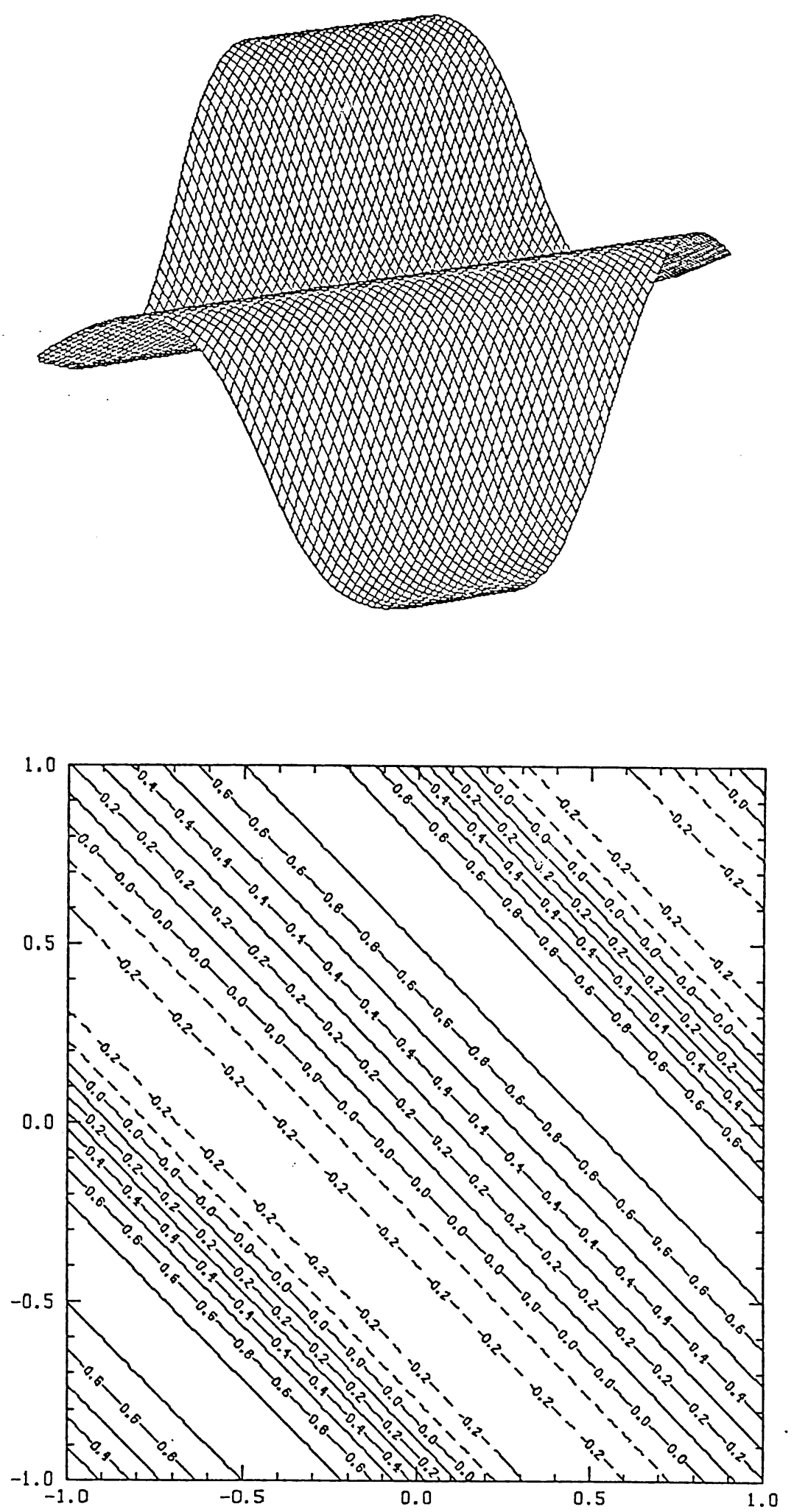

Fig. 3: The approximate solution. Initial value problem (5.1), $T=0.1$. The triangulation is $\mathcal{T}_{h=1 / 16, \text { unif }}, c f l=0.21$. 

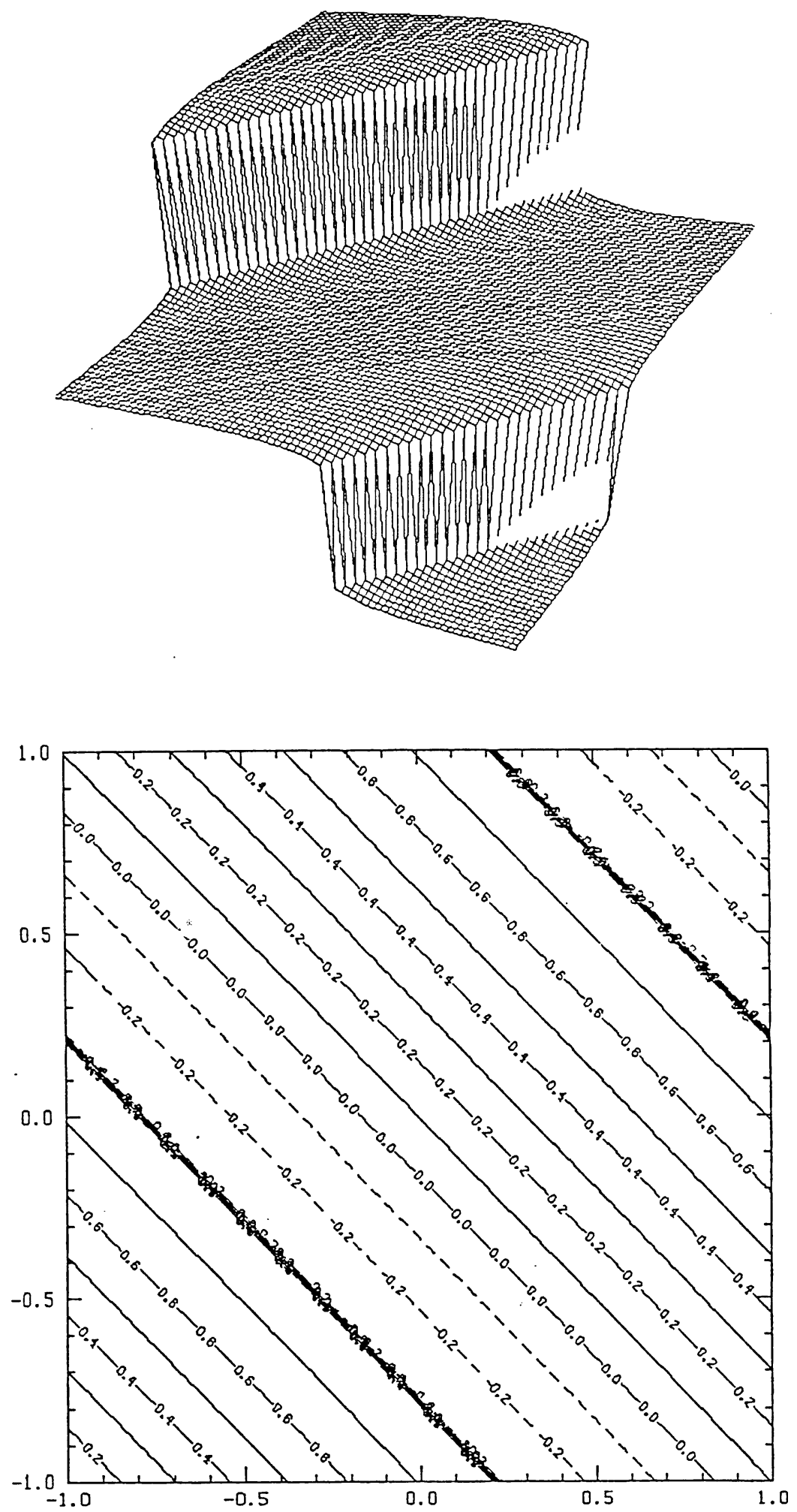

Fig. 4: The exact solution. Initial value problem (5.1), $T=0.45$. 

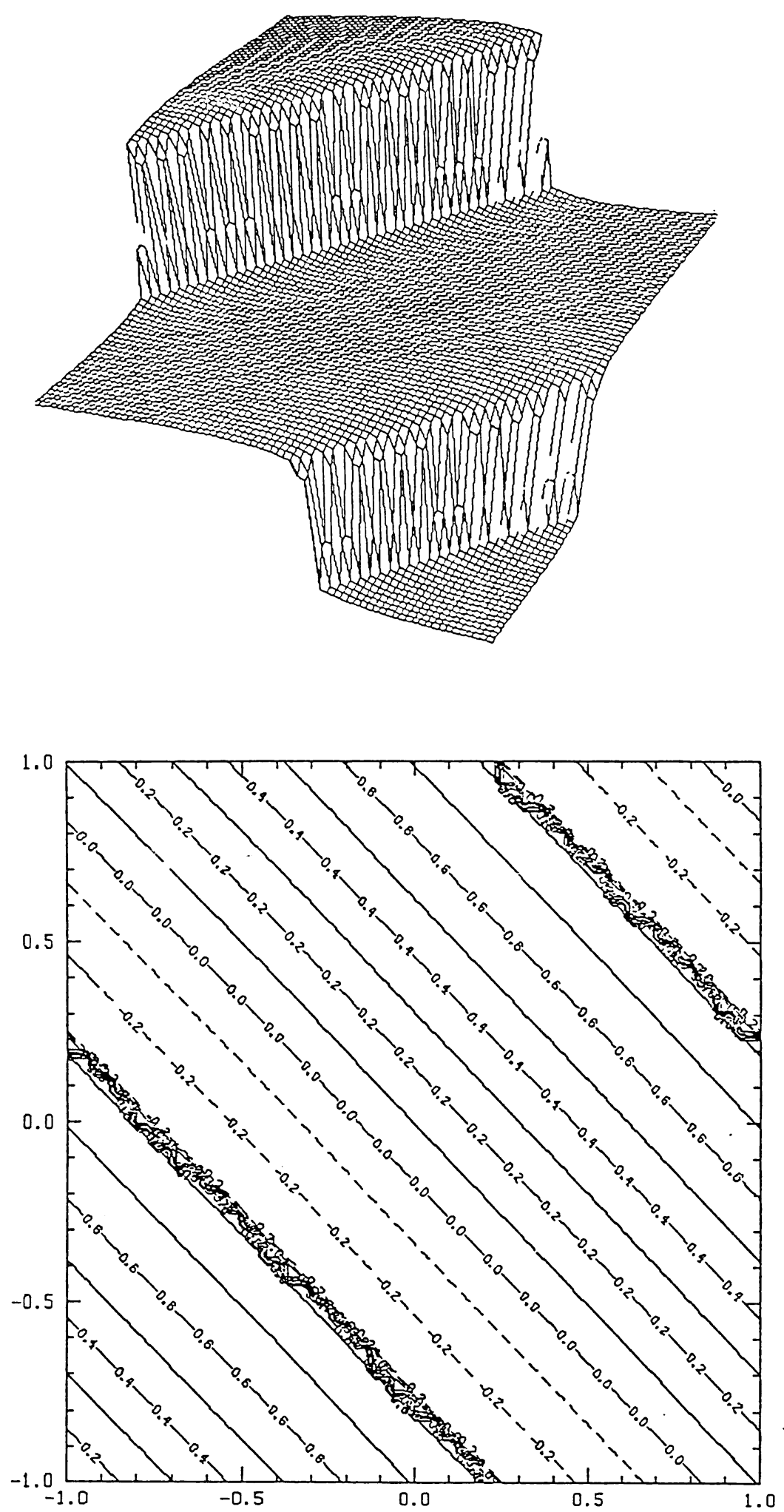

Fig. 5: The approximate solution. Initial value problem (5.1), $T=0.5$. The triangulation is $\mathcal{T}_{h=1 / 16, u n i f}, c f l=0.21$. 

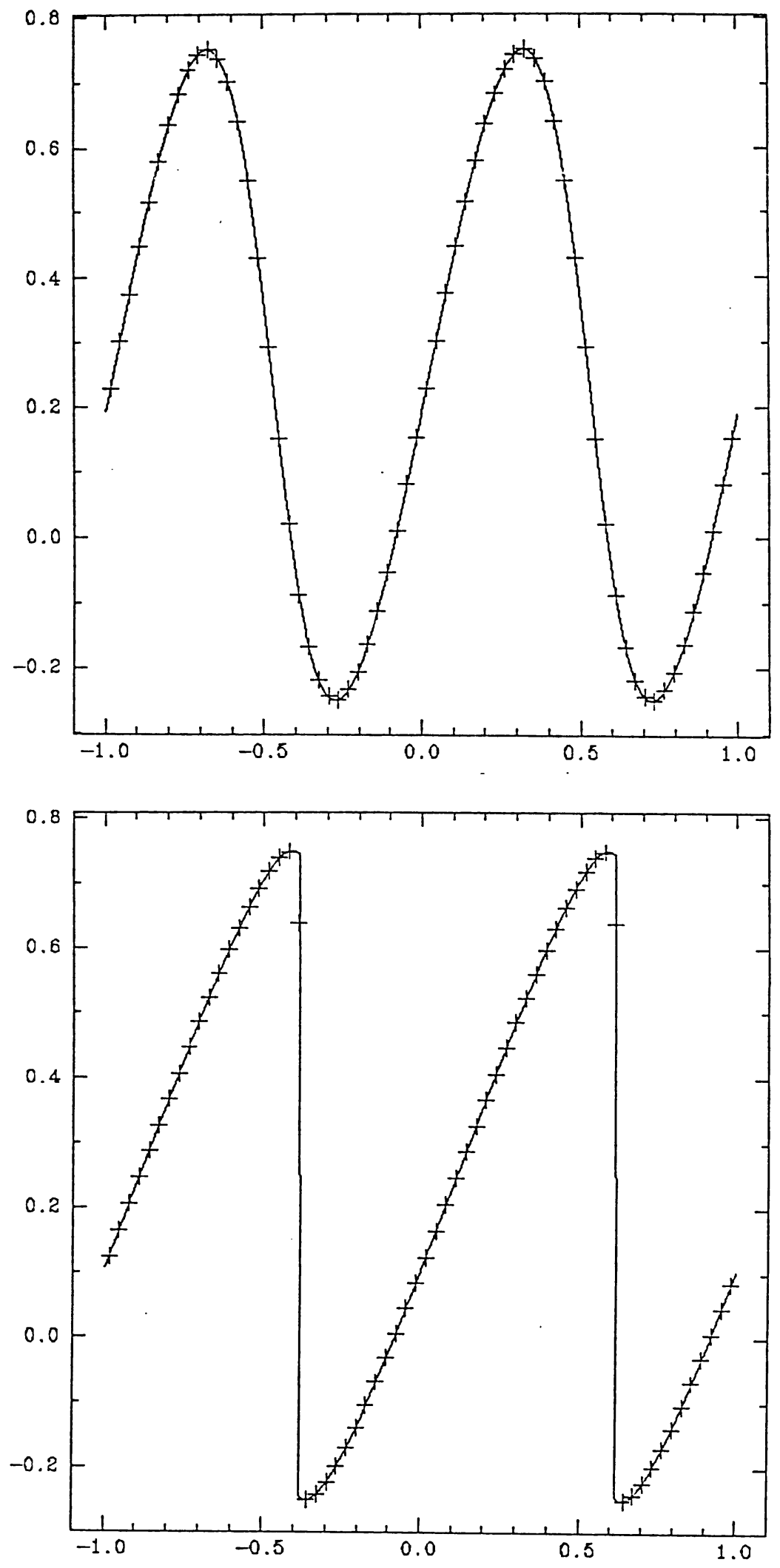

Fig. 6: Initial value problem (5.1). Cut along the diagonal. Top: $T=0.1$, bottom: $T=0.45$. 

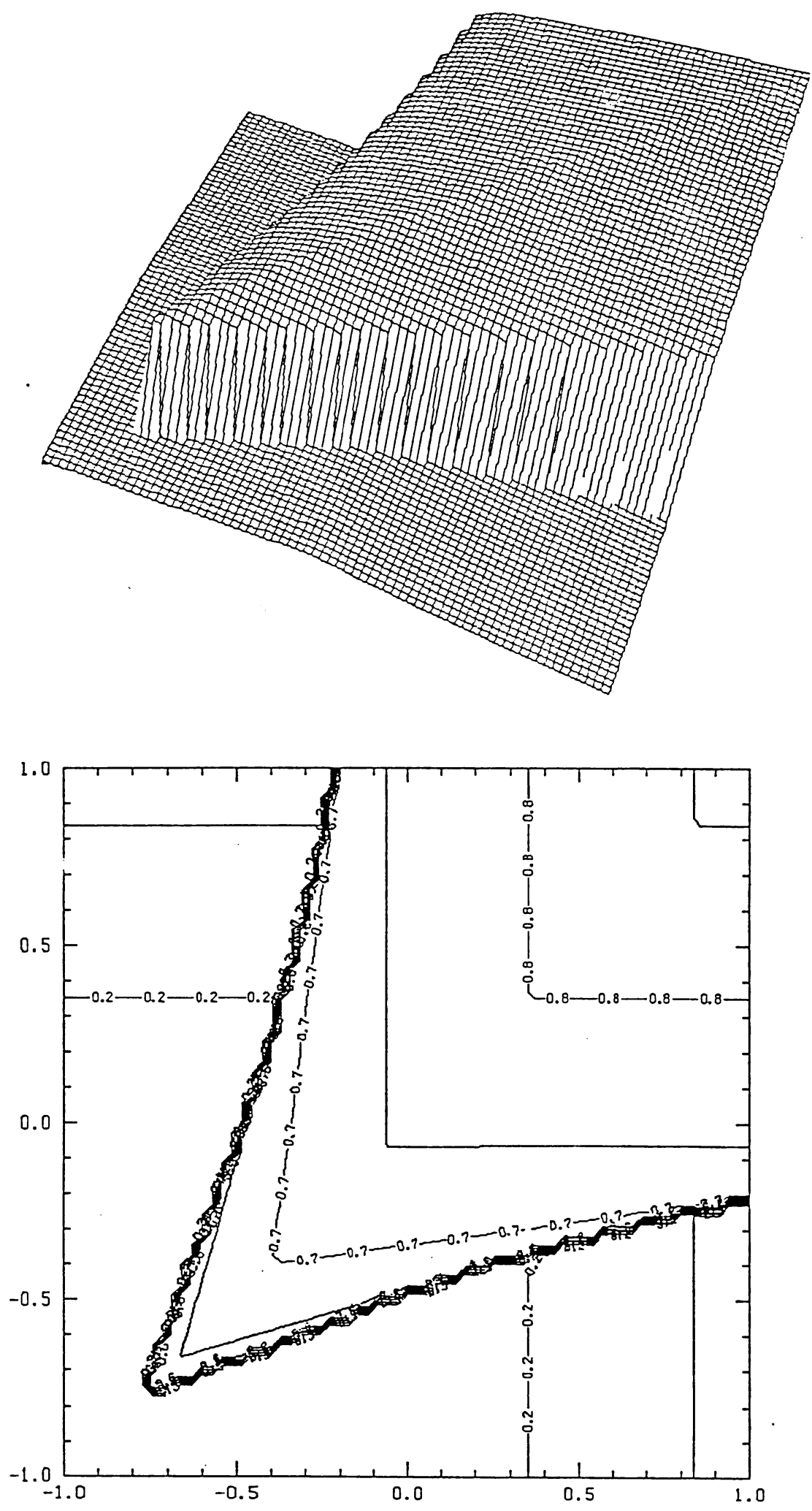

Fig. 7: The exact solution. Initial value problem (5.3), $T=1$. 

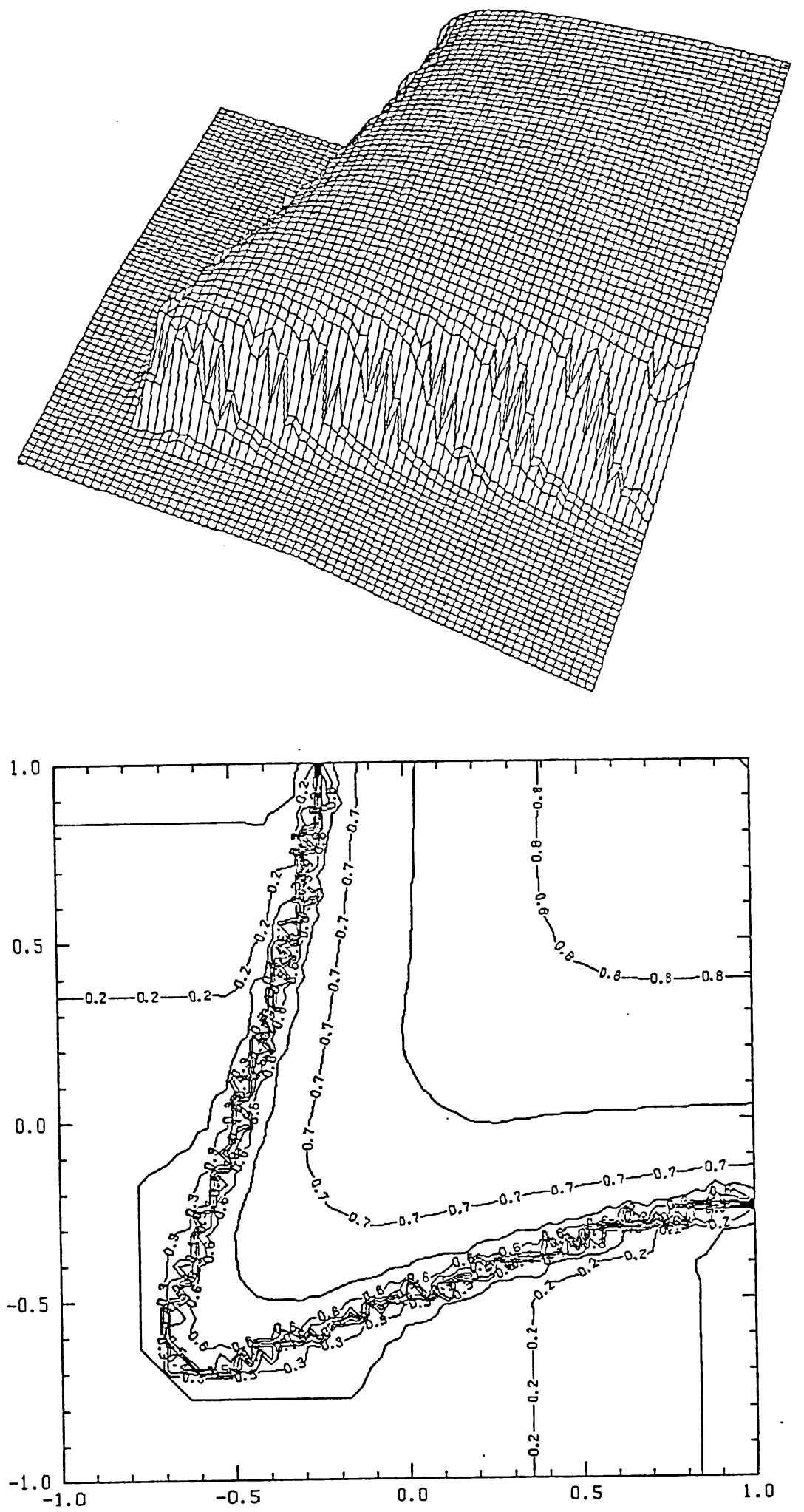

Fig. 8: The approximate solution. Initial boundary value problem (5.3), $T=1$. The triangulation is $\mathcal{T}_{h=1 / 16, \text { unif }}, c f l=0.59$. 

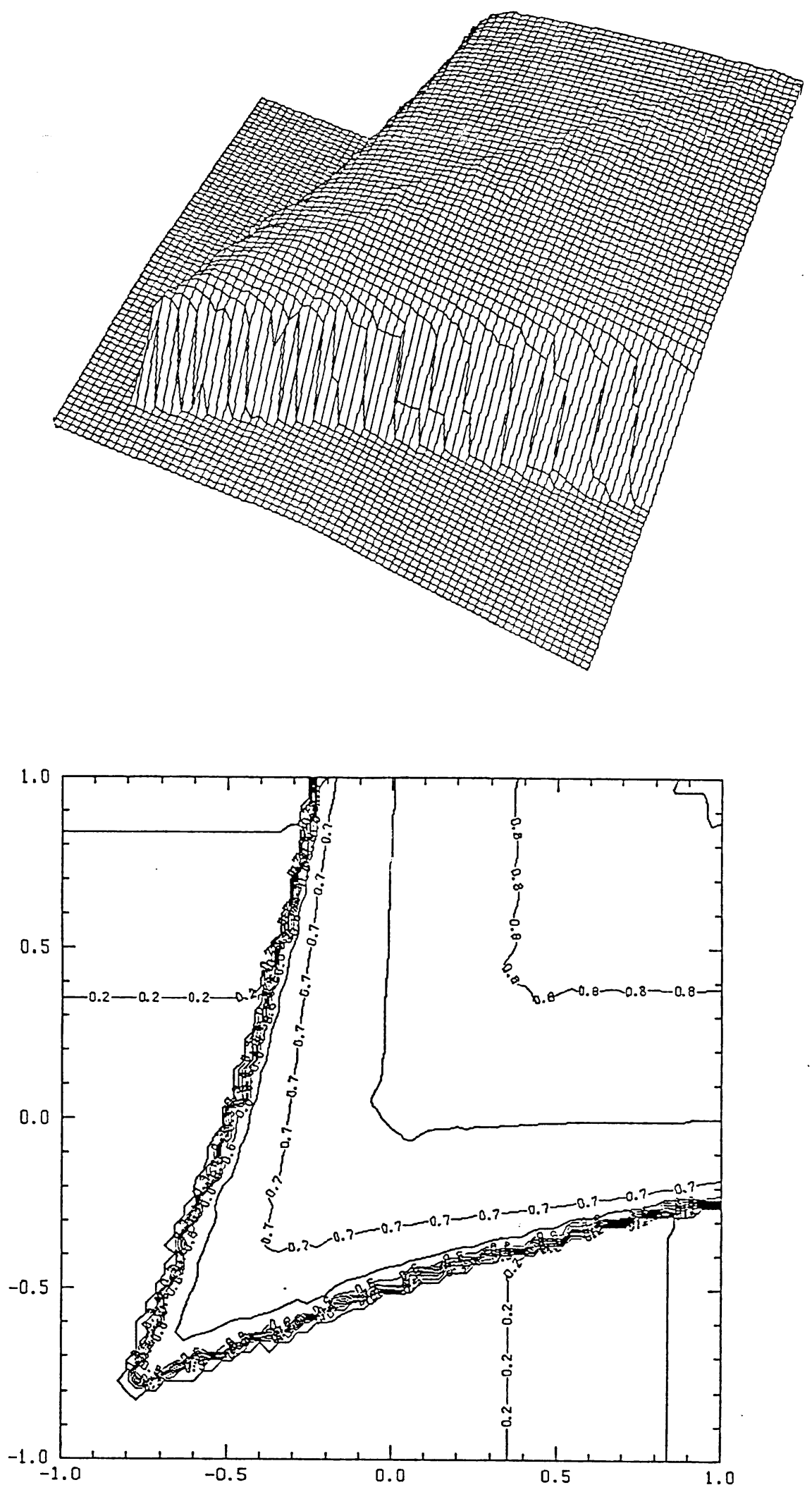

Fig. 9: The approximate solution. Initial boundary value problem (5.3), $T=1$. The triangulation is $\mathcal{T}_{h, \text { nonunif }}, c f l=0.59$. 

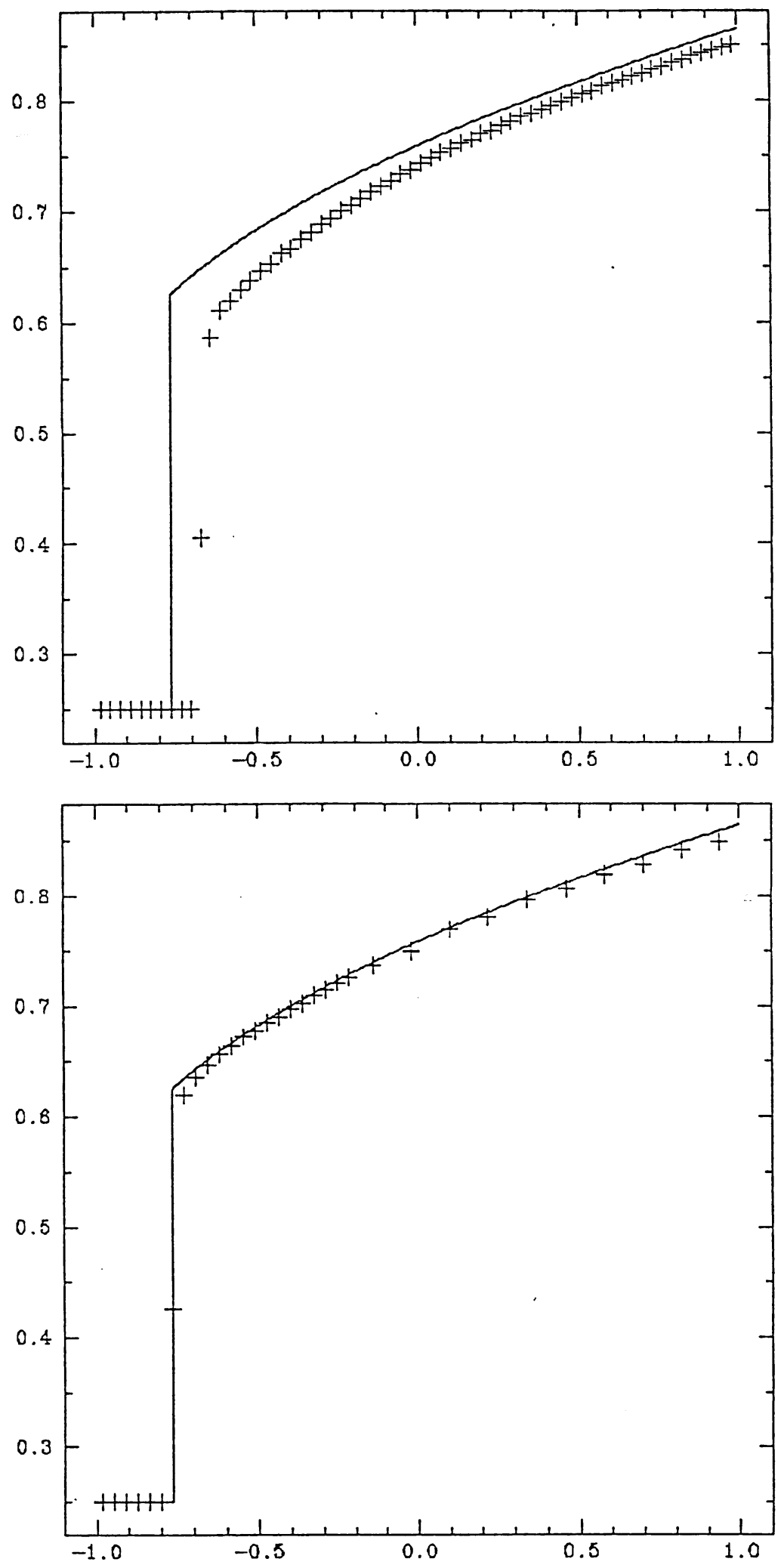

Fig. 10: Initial boundary value problem (5.3). Cut along the diagonal. Top: triangulation $\mathcal{T}_{h=1 / 16, \text { unif }}$, bottom: triangulation $\mathcal{T}_{h \text {,nonunif }}$. 
Laurent Habsieger and Dennis Stanton More Zeros of Krawtchouk Polynomials

K.M. Ramachandran Nearly Optimal Control of Queues in Heavy Traffic with Heterogeneous Servers

Y.M. Zhu and G. Yin A New Algorithm for Constrained Adaptive Array Processing

G. Yin and K.M. Ramachandran A Differential Delay Equation with Wideband Noise Perturbations

Chaitan P. Gupta Integral Type Asymptotic Conditions for the Solvability of a Periodic Fourth Order Boundary Value Problem

Chaitan P. Gupta A Two-Point Boundary Value Problem of Dirichlet Type with Resonance at Infinitely Many Eigenvalues

Jong-Shenq Guo On the Quenching Behavior of a Semilinear Parabolic Equation

Bei Hu A Quasi-Variational Inequality Arising in Elastohydrodynamics

E. Somersalo, G. Beylkin, R. Burridge and M. Cheney Inverse Scattering Problem for the Schrödinger Equation in Three Dimensions: Connections Between Exact and Approximate Methods

J.H. Dinitz and D.R. Stinson Some New Perfect One-Factorizations from Starters in Finite Fields

Albert Fässler and René Jeanneret Optimum Filter for DC/AC-Converter in Electronics

Paul Lemke On the Question of Obtaining Optimal Partitions of Point Sets in $E^{d}$ with Hyperplane Cuts

Paul Lemke and Michael Werman On the Complexity of Inverting the Autocorrelation Function of a Finite Integer Sequence, and the Problem of Locating $n$ Points on a Line, Given the $\left(\begin{array}{l}n \\ 2\end{array}\right)$ Unlabelled Distances Between Them

Chris J. Budd, Avner Friedman, Bryce McLeod and Adam A. Wheeler The Space Charge Problem Jerrold R. Griggs, Daniel J. Kleitman and Aditya Shastri Spanning Trees With Many Leaves in Cubic Graphs

Akos Seress On $\lambda$-designs with $\lambda=2 P$

Chjan C. Lim Quasi-periodic Dynamics of Desingularized Vortex Models

Chjan C. Lim On Singular Hamiltonians: The Existence of Quasi-periodic Solutions and Nonlinear Stability

Eugene Fabes, Mitchell Luskin and George R. Sell Construction of Inertial Manifolds by Elliptic Regularization

Matthew Witten A Quantitative Model for Lifespan Curves

Jay A. Wood Self-Orthogonal Codes and The topology of Spinor Group

Avner Friedman and Miguel A. Herrero, A Nonlinear Nonlocal Wave Equation Arising in Combustion Theory

Avner Friedman and Victor Isakov, On the Uniqueness in the Inverse Conductivity Problem with One Measurement

Yisong Yang Existence, Regularity, and Asymptotic Behavior of the Solutions to the Ginzburg-Landau Equations on $\mathbf{R}^{3}$

Chjan. C. Lim On Symplectic Tree Graphs

Wilhelm I. Fushchich, Ivan Krivsky and Vladimir Simulik, On Vector and Pseudovector Lagrangians for Electromagnetic Field

Wilhelm I. Fushchich, Exact Solutions of Multidimensional Nonlinear Dirac's and Schrödinger's Equations

Wilhelm I. Fushchich and Renat Zhdanov, On Some New Exact Solutions of Nonlinear.D'Allembert and Hamilton Equations

Brian A. Coomes, The Lorenz System Does Not Have a Polynomial Flow

J.W. Helton and N.J. Young, Approximation of Hankel Operators: Truncation Error in an $H^{\infty}$ Design Method

Gregory Ammar and Paul Gader, A Variant of the Gohberg-Semencul Formula Involving Circulant Matrices

R.L. Fosdick and G.P. MacSithigh, Minimization in Nonlinear Elasticity Theory for Bodies Reinforced with Inextensible Cords

Fernando Reitich, Rapidly Stretching Plastic Jets: The Linearized Problem

Francisco Bernis and Avner Friedman, Higher Order Nonlinear Degenerate Parabolic Equations

Xinfu Chen and Avner Friedman, Maxwell's Equations in a Periodic Structure

Avner Friedman and Michael Vogelius Determining Cracks by Boundary Measurements

Yuji Kodama and John Gibbons, A Method for Solving the Dispersionless KP Hierarchy and its Exact Solutions II

Yuji Kodama, Exact Solutions of Hydrodynamic Type Equations Having Infinitely Many Conserved Densities

Robert Carroll, Some Forced Nonlinear Equations and the Time Evolution of Spectral Data

Chjan. C. Lim Spanning Binary Trees, Symplectic Matrices, and Canonical Transformations for Classical N-body Problems 


\section{Recent IMA Preprints (Continued)}

Title

Matthew Witten and Caleb E. Finch, Re-Examining The Gompertzian Model of Aging Bei Hu, A Free Boundary Problem for a Hamilton-Jacobi Equation Arising in Ions Etching T.C. Hu, Victor Klee and David Larman, Optimization of Globally Convex Functions Pierre Goossens, Shellings of Tilings

D. David, D. D. Holm, and M.V. Tratnik, Integrable and Chaotic Polarization Dynamics in Nonlinear Optical Beams

D. David, D.D. Holm and M.V. Tratnik, Horseshoe Chaos in a Periodically Perturbed Polarized Optical Beam

Laurent Habsieger, Linear Recurrent Sequences and Irrationality Measures

Laurent Habsieger, MacDonald Conjectures and The Selberg Integral

David Kinderlehrer and Giorgio Vergara-Caffarelli, The Relaxation of Functionals with Surface Energies

Richard James and David Kinderlehrer, Theory of Diffusionless Phase Transitions

David Kinderlehrer, Recent Developments in Liquid Crystal Theory

Niky Kamran and Peter J. Olver, Equivalence of Higher Order Lagrangians 1. Formulation and Reduction

Lucas Hsu, Niky Kamran and Peter J. Olver, Equivalence of Higher Order Lagrangians II. The Cartan Form for Particle Lagrangians

D.J. Kaup and Peter J. Olver, Quantization of BiHamiltonian Systems

Metin Arik, Fahrünisa Neyzi, Yavuz Nutku, Peter J. Olver and John M. Verosky Multi-Hamiltonian Structure of the Born-Infeld Equation

David H. Wagner, Detonation Waves and Deflagration Waves in the One Dimensional ZND Model for High Mach Number Combustion

Jerrold R. Griggs and Daniel J. Kleitman, Minimum Cutsets for an Element of a Boolean Lattice

Dieter Jungnickel, On Affine Difference Sets

Pierre Leroux, Reduced Matrices and q-log Concavity Properties of q-Stirling Numbers

A. Narain and Y. Kizilyalli, The Flow of Pure Vapor Undergoing Film Condensation Between Parallel Plates

Donald A. French, On the Convergence of Finite Element Approximations of a Relaxed Variational Problem

Yisong Yang, Computation, Dimensionality, and Zero Dissipation Limit of the Ginzburg-Landau Wave Equation

Jürgen Sprekels, One-Dimensional Thermomechanical Phase Transitions with Non-Convex Potentials of Ginzburg-Landau Type

Yisong Yang, A Note On Nonabelian Vortices

Yisong Yang, On the Abelian Higgs Models with Sources

Chjan. C. Lim, Existence of Kam Tori in the Phase Space of Vortex Systems

John Weiss, Bäcklund Transformations and the Painlevé Property

Pu Fu-cho and D.H. Sattinger, The Yang-Baxter Equation for Integrable Systems

E. Bruce Pitman and David G. Schaeffer, Instability and Ill-Posedness in Granular Flow

Brian A. Coomes, Polynomial Flows on $\mathbb{C}^{n *}$

Bernardo Cockburn, Suchung Hou and Chi-Wang Shu, The Runge-Kutta Local Projection Discontinuous Galerkin Finite Element Method for Conservation Laws IV: The Multidimensional Case

Peter J. Olver, Invariant Theory, Equivalence Problems, and the Calculus of Variations

Daniel D. Joseph and Thomas S. Lundgren with an appendix by R. Jackson and D.A. Saville, Ensemble Averaged and Mixture Theory Equations

P. Singh, Ph. Caussignac, A. Fortes, D.D. Joseph and T. Lundgren, Stability of Periodic Arrays of Cylinders Across the Stream by Direct Simulation

Daniel D. Joseph, Generalization of the Foscolo-Gibilaro Analysis of Dynamic Waves

A. Narain and D.D. Joseph, Note on the Balance of Energy at a Phase Change Interface

Daniel D. Joseph, Remarks on inertial radii, persistent normal stresses, secondary motions, and non-elastic extensional viscosities

D. D. Joseph, Mathematical Problems Associated with the Elasticity of Liquids

Henry C. Simpson and Scott J. Spector, Some Necessary Conditions at an Internal Boundary for Minimizers in Finite Elasticity

Peter Gritzmann and Victor Klee, On the 0-1 Maximization of Positive Definite Quadratic Forms 